\title{
Gust load alleviation for a long-range aircraft with and without anticipation
}

\author{
Nicolas Fezans • Hans-Dieter Joos • \\ Christoph Deiler
}

ACCEPTED MANUSCRIPT

Please refer to the final paper:https://doi.org/10.1007/s13272-019-00362-9

Received: 17 Nov. 2017 / Revised: 09 Apr. 2018 / Accepted: 07 May 2018 / First Online: 29 Jan. 2019.

\begin{abstract}
This paper presents an overview of the DLR activities on active load alleviation in the CleanSky Smart Fixed Wing Aircraft project. The investigations followed two main research directions: the multi-objective, multimodel, structured controller design for the feedback load alleviation part and the use of Doppler LIDAR technologies for gust/turbulence anticipation. On this latter topic, the prior work made in the AWIATOR European FP6 project constituted a reference in terms of demonstrations and the objective was not to repeat these previous investigations with a real sensor in flight test but to develop new ideas for the exploitation of the Doppler LIDAR measurements for gust alleviation purposes. Very fruitful exchanges between industry partners and research organizations took place during this project and all the work presented in this paper has been made using a generic long-range benchmark provided by Airbus on the basis of the XRF-1 model.
\end{abstract}

Keywords Gust load alleviation · Multi-objective controller design · Doppler LIDAR · Feedforward load alleviation

\footnotetext{
Nicolas Fezans

DLR, Institute of Flight Systems, Lilienthalplatz 7, 38108 Braunschweig, Germany

Tel.: +49-531-295-2653

Fax: +49-531-295-2845

E-mail: nicolas.fezans@dlr.de

Hans-Dieter Joos

DLR, Institute of System Dynamics and Control, Münchener Straße 20, 82234 Weßling, Germany

Christoph Deiler

DLR, Institute of Flight Systems, Lilienthalplatz 7, 38108 Braunschweig, Germany
} 


\section{Nomenclature}

Abbreviations

ALC Active Load Control(ler)

ALDCS Active Lift Distribution Control System, active load alleviation system developed for the Lockheed C5-A

AWIATOR Aircraft Wing Advanced Technology Operation, European FP6 project investigating many innovative technologies for future and more efficient aircraft

BFGS Broyden-Fletcher-Goldfard-Shanno, a well-known quasi-Newton optimization algorithm

$\mathrm{C}^{*}, \mathrm{C}^{*} \mathrm{U} \quad$ Control concepts in the pitch axis based on the blending of the load factor and the pitch rate (with airspeed feedback for $\mathrm{C}^{*} \mathrm{U}$ )

DELICAT DEmonstration of LIdar based CAT detection, European FP7 project on the detection of clear air turbulence

DLC Direct Lift Control, control surfaces/effectors permitting to directly control the aircraft lift (i.e. not through variations of the angle of attack)

DLR Deutsches Zentrum für Luft- und Raumfahrt (German Aerospace Center)

EFCS Electronic Flight Control System

FBALC Feedback Active Load Controller, name of the feedback part of the herein proposed load alleviation functions

FOWT Fast Orthogonal Wavelet Transform

FP6 Sixth Framework Programme, European Union's Research and Innovation funding programme for the period 2002-2006

FP7 Seventh Framework Programme, European Union's Research and Innovation funding programme for the period 2007-2013

GCS Gust Control System

GLAS Gust Load Alleviation System

GN Gauss-Newton, optimization algorithm optimized for nonlinear least squares problems

HR HTP root

HTP Horizontal Tailplane

IRS Inertial Reference System

LARS Load Alleviation and Ride Smoothing

LIDAR LIght Detection And Ranging

LQR Linear-Quadratic Regulator

OLGA Open Loop Gust Alleviation

pdf Probability density function

$\mathrm{RCAH} \quad$ Rate Command Attitude Hold

RMS Root Mean Square

SFWA Smart Fixed Wing Aircraft, Integrated Technology Demonstrator (ITD) from the European CleanSky project

WR Wing root

XRF-1 Generic long-range aircraft model designed by Airbus 


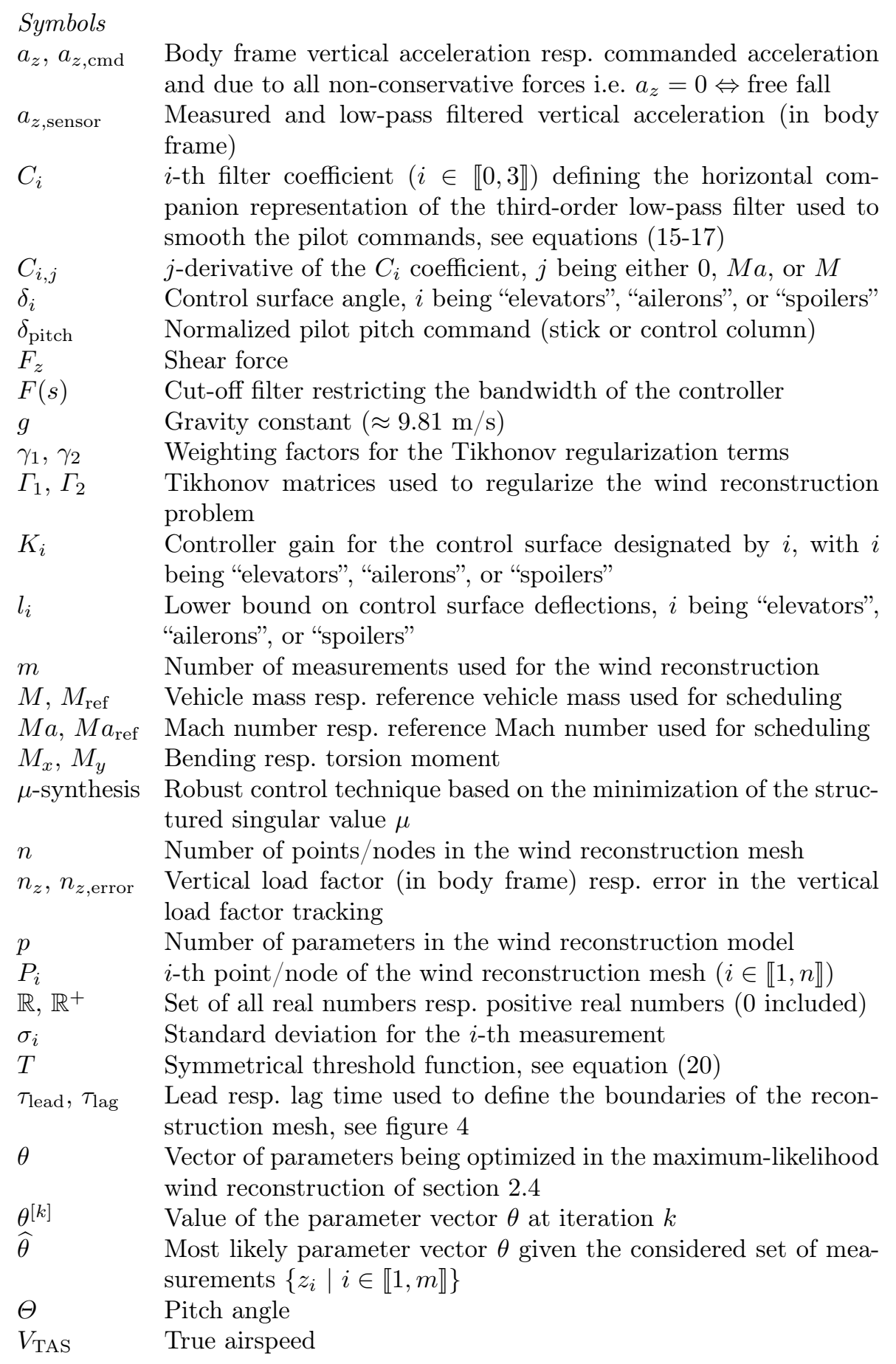


$z_{i}, y_{i}(\tilde{\theta}) \quad$ Measurements used for the wind reconstruction resp. corresponding model outputs for given values $\tilde{\theta}$ of the parameter vector

\section{Introduction}

Inhomogeneous wind fields such as turbulence and gusts are causing variations of the global and local aerodynamic forces and moments that are applied to the aircraft structure. In addition to causing structural loads that the structure should be designed to handle, these additional forces and moments also cause passenger discomfort and anxiety. Active load alleviation of gusts and turbulence is not a new topic: the investigations made on active load control to solve the Lockheed C-5A fatigue issues and leading to the development of the "Active Lift Distribution Control System" (ALDCS) dates back forty years [17]. Already at that time, the trade-off between structure mass and use of active control technologies was present. Historically, within the last forty years, there have been two main drivers for investigations on active load alleviation:

- either a structure design was available, but was for some reasons too weak and the use of active control solved (or was meant to solve) the problem,

- or designers were interested in increasing the efficiency through mass savings thanks to load reductions.

Numerous load alleviation functions have been successfully implemented, for instance on the following airplanes: Lockheed C-5A, Lockheed L-1011500, Boeing B-1, Northrop Grumman B-2, Airbus A320, Airbus A330/A340, Airbus A380, Boeing 787, Airbus A350. [42] and the references therein give an interesting overview of the applications of active gust alleviation.

The numerous successes of active control technologies for airplane gust alleviation logically ended up reaching even the maximum technology readiness level (TRL) of 9 for some of these systems. Consequently, the orientation of the research activities of DLR on gust alleviation moved from more classical gust alleviation system design (such as in OLGA $[24,33,4]$ or LARS $[30,16,31]$ ) to the investigation of more advanced solutions for an even improved alleviation performance. The investigations presented hereafter combine two main ideas:

1. better anticipation capability of future loads for feedforward load alleviation

2. improved controller synthesis methods for multi-objective and robust feedback load alleviation $[26,27,29,28,36,35]$.

Previous work had been performed in these directions, especially during the AWIATOR project, and had lead in particular to the GCS [19] and GLAS $[15,18,20]$ systems and to consider also the use of Doppler LIDAR (LIght Detection And Ranging) sensors for load alleviation purposes (in cooperation with the other project partners) $[19,15,18,20,43,41]$. As for these previous investigations, the feedforward load alleviation function presented hereafter is based on the idea that with a better anticipation of the near future loads a 
higher load alleviation performance can be achieved. Consequently, in all these systems one of the major components is dedicated to the determination of the expected near future loads. This is realized by gathering information on the wind field ahead of the aircraft, which in the current concept is based on a Doppler LIDAR sensor and a rather extensive processing of the measurements. This information is then used to alleviate (in feedforward) these future loads.

Anticipating the future loads opens new possibilities in terms of load alleviation, but cannot replace a feedback controller that directly acts on the closed-loop behavior of the structural modes. These two parts are radically different and complementary. Therefore the present work also includes investigations on load alleviation with a feedback scheme. Flight control law design in general and active load control function (ALC) design in particular are multi-variable control problems where various strict requirements have to be satisfied. In order to cope with uncertainties, missing or erroneous feedback or scheduling variables robustness of the controller is indispensable. To tackle these problems, an optimization-based multi-objective synthesis approach is proposed $[26,27,36,35,32]$. Whilst, traditionally, the design of ALC-functions is based on linear flexible aircraft models, see for instance $[32,25]$, the multiobjective optimization-based approach is able to handle nonlinear flexible aircraft models augmented by nonlinear flight control systems (e.g. due to deadzone, saturations, other nonlinearities, or even containing pure delays).

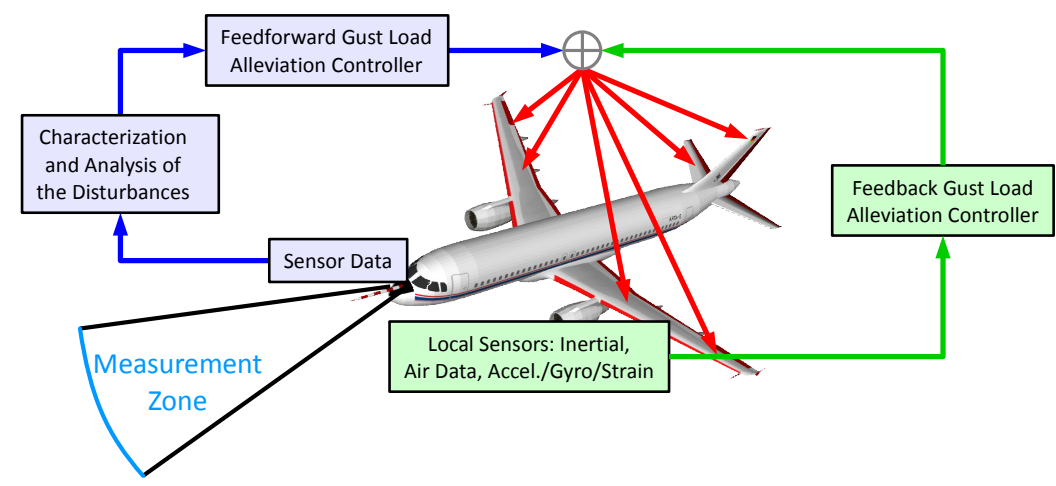

Fig. 1: Schematic representation of the feedforward load alleviation principle.

The proposed system architecture consists of a "classical" feedback controller based on the inertial measurements (and if available direct measurements on the structure) and a feedforward controller based on remote wind measurements (see figure 1). Each of both components has capabilities that the other cannot provide (anticipation can only be provided by the feedforward function, whereas modification of the internal dynamics can only be provided by the feedback function). Combining both functions allows a greater load alleviation performance than each function could achieve by itself. 
Figure 2 presents the high-level structure of the whole flight control system. The feedback load alleviation function can be seen as an add-on to the regular control laws (later referred to as "EFCS"), which provide the flight control augmentation function ( $n_{z}$-law / $\mathrm{C}^{*} / \mathrm{C}^{*} \mathrm{U} / \mathrm{RCAH}$ etc.). These "regular" laws can be developed very early in the design process and are the main drivers for the handling qualities of the aircraft. The other functions (such as feedback and feedforward gust and turbulence load alleviation) are in general trying to satisfy other criteria without deteriorating the handling qualities that are provided by the "regular" laws. The current work focuses only on the feedback and on the feedforward load alleviation functions. Note that in this figure various possible interconnections of the feedforward module with the rest of the system are shown. Not all of these interconnections are always required: the need for each one of them depends on the exact behaviors of the various controllers and thereby on the undesired interactions that might have to be prevented.

Structural loads in an airplane are not only generated by gusts and turbulence but can be caused (among others) by maneuvers or during touchdown and ground operations. In order to optimize weight savings various load cases might need to be considered simultaneously. The focus of this paper is on gust load alleviation and no maneuver load alleviation function is shown hereafter. Note, however, that a maneuver load alleviation function could easily be added to the active load alleviation functions presented hereafter and the fact that the functions shown hereafter are designed such that they do not deteriorate the maneuverability of the aircraft will be shown.

Section 2 presents the Doppler LIDAR feedforward control architecture. An explicit wind reconstruction is performed online and is then exploited using a combination of a time-frequency decomposition of the future vertical wind and a decentralized control scheme. This unusual approach permits to easily satisfy the strong and nonlinear control allocation objectives that were specified by the industry partners during the CleanSky Smart Fixed Wing Aircraft research project. Section 3 presents the design of the feedback load alleviation function. The control design methodology used is based on the formulation of the design problem as a multi-objective optimization problem. Finally, section 4 presents the results obtained from the application to a large and flexible transport airplane benchmark model (derived from the XRF-1 model data originally provided by Airbus and integrated in an aeroelastics / flight dynamics model by DLR). The results include the load alleviation performance along the wing and along the horizontal tailplane (HTP) as well as other indicators (e.g. passenger comfort).

\section{LIDAR-Based Feedforward Load Alleviation Controller}

In this section, the feedforward load alleviation controller is presented. First, section 2.1 presents shortly the Doppler LIDAR sensor measurements that are used by the feedforward controller. These measurements are not directly 


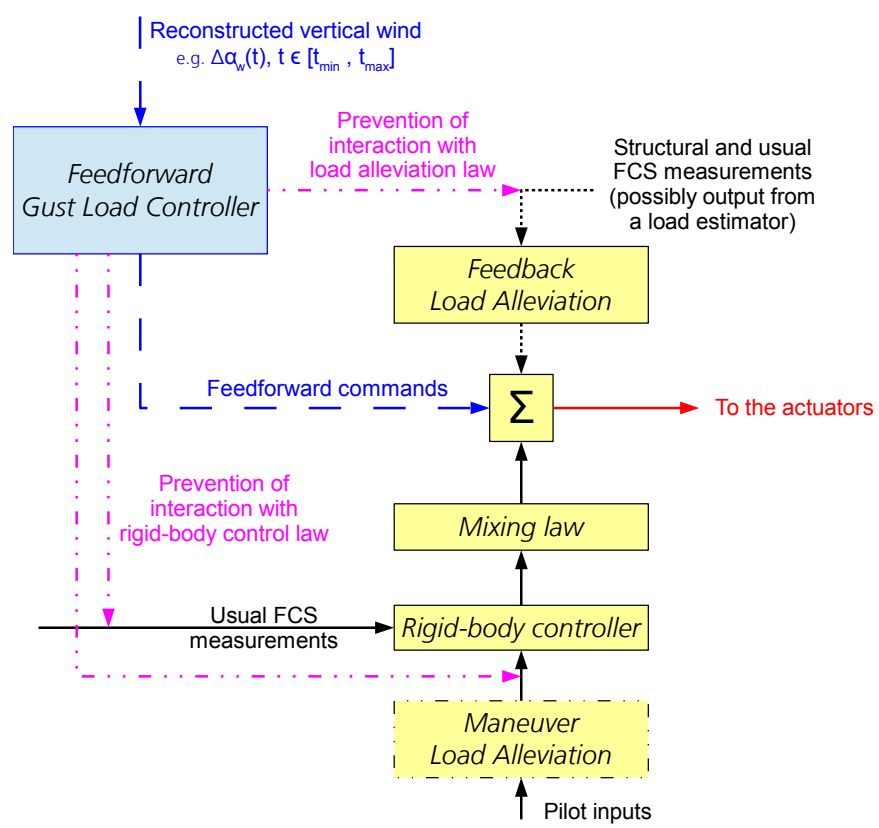

Fig. 2: Generic flight control system architecture

well-suited for use in a feedforward controller and require a wind reconstruction process in several steps which are detailed in sections 2.2-2.6. Section 2.7 presents the general concept underlying the feedforward controller that exploits this reconstructed wind information. The implementation details for the feedforward controller can be found in [8].

\subsection{Remote Wind Sensing with Doppler LIDAR}

The basic idea for the gust load alleviation using a Doppler LIDAR sensor is to measure the atmospheric disturbances before they reach the wings of the aircraft and induce additional loads on the aircraft structure. By measuring these disturbances in advance, the load alleviation function can anticipate the future loads and begin counteracting them before having encountered the disturbances that will cause them. This corresponds to the left part of the schematic representation shown in figure 1 . The time delay between the measurement and the encounter gust-wings is approximately the distance between the measurement position and the wings divided by the true airspeed. On a typical airliner, the lead time of a measurement at the aircraft nose is small and, as a consequence, the anticipation capability of a feedforward based on these sensors is very restricted. The use of a Doppler LIDAR permits to measure the wind further ahead of the aircraft nose (typically $60-300 \mathrm{~m}$ ) and thereby to better anticipate the coming gusts and turbulence. 
As indicated through its name, a Doppler LIDAR makes use of the wellknown Doppler effect, which basically consists in a frequency shift of any observed wave when emitter and receiver (i.e. observer) of the wave are moving with respect to each other. The idea is to measure the disturbance shortly before encountering it and to counteract it: this type of LIDAR should not be confused with LIDAR systems that aim at detecting the presence of turbulence well in advance (several kilometers) in order to avoid the area. This other type of LIDAR systems was for instance investigated in the DELICAT European project $[47,48]$ and further explanations on the different types of forward-pointing LIDAR systems can be found in [46]. For the sake of simplifying the explanations, only a so-called direct-detection pulsed Doppler LIDAR $[43,41,46,22,5,21,49]$ is considered hereafter. Nevertheless, the measurement processing approach and feedforward strategy could easily be adapted to other types/variants of remote wind sensors. In the considered case, a short pulse (typically lasting for a few tens of nanoseconds) of laser light is emitted. The laser beam has a very low divergence, which allows to illuminate only a specific area ahead of the aircraft. The pulse of light advances along in the laser direction and at each location a tiny fraction of this pulse is scattered by the molecules of the air (and possibly some aerosols if present). The scattering occurs in all / a wide range of directions and part of the scattered light goes back to the LIDAR sensor. The frequency of the light that is scattered back to the LIDAR sensor can be compared to the one of the light that has been emitted. A shift toward higher frequencies (so-called blue shift) signifies that the sensor and the aerosols/molecules that scattered the light back were moving toward each other. On the contrary, if they are moving away from each other then the wave will be shifted towards lower frequencies (red shift).

If the presence of aerosols can be assumed, so-called coherent heterodyne detection principles with a laser source in the infrared domain are generally the best choice. However, for the alleviation of clear air turbulence at high altitudes (possible lack of sufficient aerosol concentration) and if a high availability of the remote wind measurement is desired, a so-called direct detection principle with a laser source in the ultraviolet domain can be used, since it can work with the so-called Rayleigh-scattering on the molecules of the air. More information on the remote wind measurement technologies and their respective capabilities can be found in the literature, for instance in $[49,23,43,41,2$, $3,40]$ and references therein.

Ideally the entire wind field ahead of the aircraft would be perfectly known: all three wind components, at every location, and with no measurement error. Due to the fact that the airplane flies at a high velocity and that the bandwidth of the flight control system is limited, a spatial resolution along the flight path higher than 4 to 7 meters is not required. Only the wind information in the close vicinity of the airplane trajectory is required, which with the typical measurement distances $(60-300 \mathrm{~m})$ represents only a few degrees in terms of field of view. In terms of wind velocity, the most important component for loads is the vertical component, since this component has the greatest influence on the local lift (via a modification of the angle of attack). The lateral component 
is only secondary for load alleviation purposes and finally the longitudinal component (i.e. in flight path direction) has an effect on the lift through a change of the airspeed, but this effect is assumed to be relatively negligible.

When using Doppler LIDAR sensors, only the relative wind component in the direction of the laser beam is measured. If the wind is measured at locations ahead of the aircraft, then the laser beam (also called line-of-sight) direction is almost collinear with the flight path. With other words, the sensor readings are measuring the least interesting velocity component of the wind (basically the true airspeed) and not the interesting vertical and lateral components.

A way to estimate or reconstruct the missing information (other velocity components as well as the wind at locations that were not directly measured) is to measure the wind at locations with various vertical and lateral offsets with respect to the airplane flight path. The resulting line-of-sight directions are not (all) collinear anymore and the analysis of the differences between the different sensor readings permits to estimate the transversal components (lateral and vertical) of the wind. This method assumes implicitly that the wind is homogeneous between the points where the measurements are made. This assumption is of course difficult to validate and strongly depends on the current atmospheric conditions encountered by the aircraft. The closer the measurements are located, the more likely it is that this assumption is somewhat valid. Reducing the distance (laterally and vertically) between the measurements would however lead to reduce the angles between the different line-of-sight directions, eventually leading to very small differences between the sensor line-of-sight velocities that are measured. This has a major drawback because these measurements cannot be perfect (noise, biases, etc.). In particular, the signal-to-noise ratio (signal being the difference due to the wind transversal components and noise being linked to the noise on each measurement) becomes very poor when calculating the difference between measurements taken under almost collinear directions. With other words, there will necessarily be a trade-off to be made between the validity of the homogeneity assumption (linked to the distance of the measurements to the flight path) and the signal-to-noise ratio for the reconstructed transversal wind components.

\subsection{Overview of the Wind Reconstruction Algorithm}

In this section, the way a complete wind field was reconstructed from a set of line-of-sight wind measurements is presented. This wind field reconstruction step is one of the main novelties compared to previous works on gust detection and measurement based on LIDAR sensors. For instance, in the AWIATOR system [41] the processing considers only a group of four measurements and does not account for the fact that these measurements were not made simultaneously and therefore the four measurements are not located at the same distance from the aircraft (see. equations (8-9) of [41]). Taking each group of four measurements and not integrating the neighboring measurements reduces the performance of filtering/smoothing attempts with no phase lag (counter- 
acting gusts too late might increase loads instead of reducing them). All in all, the performance of the wind field reconstruction shown hereafter is significantly higher (higher precision and lower noise after reconstruction) than the one of [41]. When using a measurement processing strategy as presented hereafter, LIDAR sensor configurations that were otherwise not considered interesting (e.g. towards higher measurement repetition rate at the cost of an increased noise level on the line-of-sight velocity measurements) also become more practicable and very promising.

Figure 3 represent the whole feedforward load alleviation function. The top part of this figure (above the dashed black line) is the wind reconstruction algorithm, the lower right part symbolizes the client system (i.e. the load alleviation function shown in section 2.7), and the lower-left part represents the LIDAR sensor and the buffering of the measurements. As indicated in the light blue dashed boxed each of these part is working with its own sampling rate. The information on each measurement that are used for the proposed wind field reconstruction algorithm consists of:

- the measurement itself (line-of-sight relative velocity of the sensor with respect to the air at the measurement location)

- and the associated metadata:

- the location in a local reference system at which the measurement was made,

- the orientation of the line-of-sight direction under which the measurement location was observed,

- the inertial speed of the sensor (expressed also in the local reference system) at the time of the measurement.

The first step (lower-left part) consists in buffering the measurements (including the corresponding metadata). Depending on the content of the buffer, the main algorithm will be triggered/activated or not. This decision will in general be made based on a very simple computation, such as by defining a deviation index between the measurements and the measurements that would have resulted if the surrounding air would have been perfectly homogeneous. In that particular example, the threshold applied should be set to a higher value than the deviation that will result from the measurement noise.

If the main algorithm is started, it will determine the wind field that explains best the considered measurements. In this process, a parameterized model of the disturbance (gust, turbulence, etc.) will be used and the parameter values be searched. The model used is presented in section 2.3 and the way the parameter values are searched is presented in section 2.4.

Finally, once the main part of the wind reconstruction successfully found the "best" parameter values, the plausibility of the obtained wind field is checked prior to any use by the feedforward alleviation functions. It should be noticed that usually at least three different rates are used within the whole system: the buffering rate (synchronous with the sensor rate), the estimation update / wind reconstruction rate (usually relatively low: typically 3 to $10 \mathrm{~Hz}$ ), and the client system / alleviation function rate (usually the same rate than 


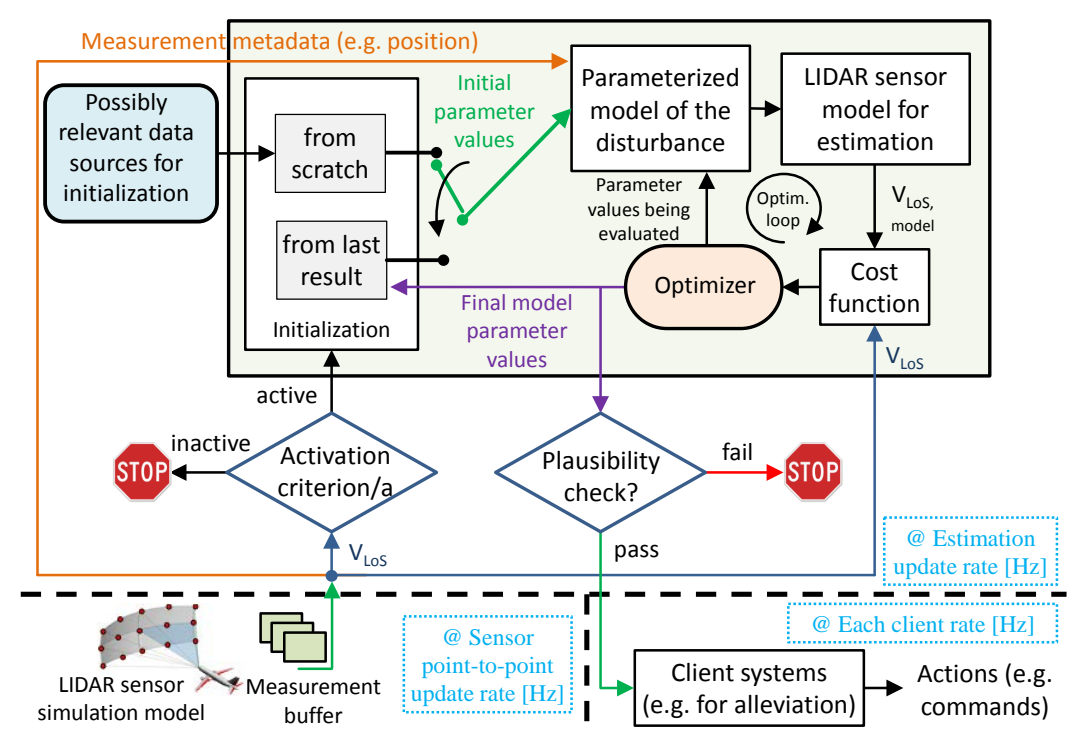

Fig. 3: General overview of the wind reconstruction algorithm.

that of the main flight control computers). The estimation update / wind reconstruction rate cannot be increased due to the required computation time. The client system (here alleviation function) rate can however be higher, because the reconstructed wind field is large enough and the aircraft will remain inside it for the time between two updates. In between, the aircraft location and orientation changed and other locations of the wind field are used for the alleviation function. As a consequence the wind reconstruction rate does not induce an upper limit (e.g. through the Nyquist-Shannon sampling theorem) on the disturbance frequencies that can be alleviated. The wind reconstruction is performed for a domain, mainly ahead of the aircraft, and is necessarily limited in size due to the fact that the LIDAR sensor itself has a limited range. The time $t_{d}$ until the aircraft reaches the end of this domain can be approximated by dividing the size of this domain in the direction of the flight path by the current true airspeed of the aircraft. The time $t_{r}$ between two updates of the wind profile (i.e. execution of the wind reconstruction algorithm) shall ideally be at least two to three times lower than $t_{d}$ in order to prevent any deterioration of the feedforward performance.

The wind reconstruction process is closely related to the process shown in [6] for the identification of wake vortices. The strong commonalities as well as the existing differences between these two applications are described in [11,12]. In the next sections, the free-form wind field model and the formulation of the maximum-likelihood wind reconstruction problem are only briefly reminded and the readers are referred to [12] for the details and for the wind reconstruction results. Significant performance improvements of the wind reconstruction algorithm could be obtained in terms of computation time compared to the 
version used in [12] by exploiting the linear least-squares structure of the problem. These improvements are shown hereafter in section 2.6.

\subsection{Free-Form Wind Field Model}

Whereas for other wind reconstruction problems analytical models of the wind field might exist and be applicable for reconstruction problems (see for instance $[14,38,7,6,11]$ and references therein), gusts and turbulence are stochastic by nature and no particular model structure and shape shall be assumed. Analytical models for gust and turbulence do exist, but are not suited for the considered wind reconstruction. Artificial gust shapes (e.g. 1-cosine) and artificial turbulence spectrums (Dryden, von Kárman) were defined for certification purposes: they can be considered as "representative" in the sense that they permit to define standardized cases for the computation of structural loads and the certification of the airplane structure. However, they do not represent a wind field shape that can be considered as similar to the real wind fields that will be encountered by the airplane and that was measured with the LIDAR. As a consequence, these models are not adequate for gust/turbulence-related wind field reconstruction.

A way to cope with the absence of adequate model forms for gust and turbulence is to use a free-form model structure. The idea is to represent the gust/turbulence wind field by a mesh where a velocity vector is set for each node of the mesh. Any wind field can in principle be represented by such a mesh, as long as enough nodes are taken. For the application to gust load alleviation, small-scale wind variations are not relevant and there is no real benefit in using a very fine mesh.

The chosen mesh is composed only of nodes placed at regular intervals along the flight path of the aircraft. The last point (the furthest ahead of the aircraft) $P_{n}$ is placed at the predicted location of the aircraft at time "now $+\tau_{\text {lead }} . "$ The first point $P_{1}$ is also located along the current flight path but behind the aircraft at the distance $\tau_{l a g} \cdot V_{T A S}$.

Figure 4 shows a graphical representation of this one-dimensional mesh. The local wind vectors associated with the nodes of the mesh are here represented by the red arrows. In the numerical implementation used, these local wind vectors are represented by their components in a North-East-Down reference frame. In this simple 1D-mesh-based model, it is implicitly assumed that the wind vectors at all locations within a plane that is perpendicular to the flight path direction are identical (which means that the wind is constant along any transversal direction). These wind vectors being all equal, they are also equal to the wind vector at the intersection between the flight path and the considered plane. When this intersection is not also defined as a node of the mesh, the wind vector at the intersection is obtained by linearly interpolating between the surrounding two nodes. Typical values permitting to estimate a wind field for load alleviation purposes are:

- Lead-time $\left(\tau_{\text {lead }}\right): 1$ to 2 seconds 


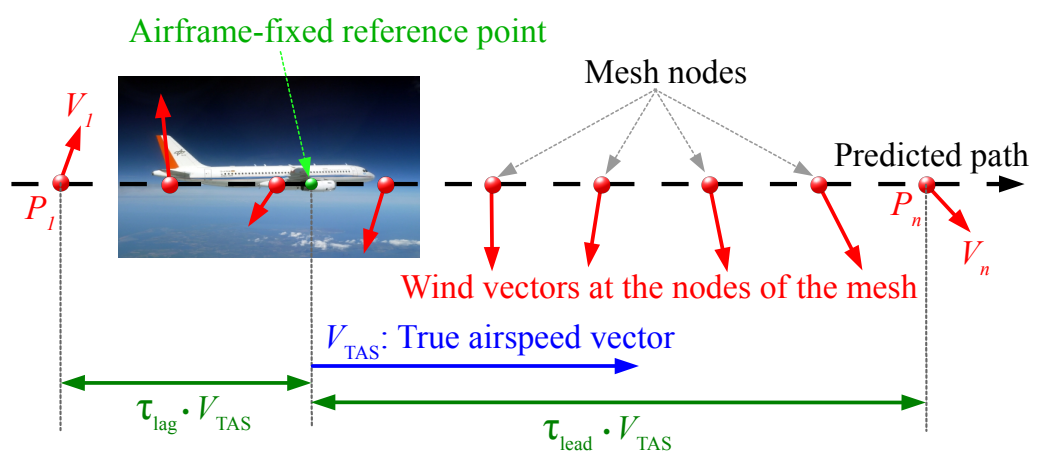

Fig. 4: Schematic representation of a 1D-mesh along the current flight path.

- Lag-time $\left(\tau_{\text {lag }}\right): 0.5$ seconds

- Number of nodes: $\approx 30$.

Further details on the way the values of these parameters should be chosen can be found in $[12,10]$. The lead and lag time values need to be scaled with the size of the considered gust lengths (usually the sizing gusts). The considered flexible long range aircraft for which these values were determined has a sizing gust length around $300 \mathrm{ft}$. If the critical gust length is not $300 \mathrm{ft}$, these times shall be scaled proportionally (i.e. for a gust of $100 \mathrm{ft}$ these times shall be divided by a factor 3 ). The same applies with the flight speed, but the scaling factors involved will usually be smaller.

\subsection{Maximum-Likelihood Wind Reconstruction and its Regularization}

The goal of the wind reconstruction is to interpret the line-of-sight wind velocity measurements made and to deduce the most likely wind field that could have caused these measurements. Indeed, only restricted information has been gathered through the measurements and the interpretation/deduction part is crucial and is described hereafter. The process described hereafter corresponds to the "optimization loop" at the top right part of the wind reconstruction process shown in figure 3 .

The measurements are usually noted with the letter $z$ and indices are used to distinguish them. Let $m$ be the number of measurements currently contained in the database (or buffer). Then, let

$$
\left\{z_{i} \mid i \in \llbracket 1, m \rrbracket\right\}
$$

be the set of measurements used during the wind reconstruction process. Let $\theta=\left[\theta_{1}, \theta_{2}, \ldots, \theta_{p}\right]$ be the vector of all $p$ wind field model parameters. For a given set of parameter values $\tilde{\theta}$ the $m$ model outputs $\left\{y_{i}(\tilde{\theta}) \mid i \in \llbracket 1, m \rrbracket\right\}$ corresponding to the measurements made (same location and conditions) can 
be computed and compared to the measurements $\left\{z_{i} \mid i \in \llbracket 1, m \rrbracket\right\}$. The closer the measurements and the corresponding model outputs, the more likely it is that both the model (i.e. its structure) and the current parameter values are right. More formally, for each measurement and each model parameter vector value, a probability density function (pdf) $x \mapsto p(x \mid \theta)$ can be used to represent the designer's belief regarding the measurement under the assumption that these model parameters are right.

For the sake of explanation, consider a simple system being a solid with a given mass $M$ whose mass will be measured using a scale and the relationship between mass and weight: $P=M g$. If the gravity field is perfectly known the measured mass is only affected by the sensor uncertainty/error of the scale. The pure sensor error usually has several sources (calibration errors, nonlinearities, quantization, etc.) and can usually be characterized. If the model expressed by the equation $P=M g$ is uncertain (e.g. the equation is approximated or the gravity field itself is uncertain), these model errors and the pure sensor errors combine to form the "measurement error." By describing the stochastic properties of each error source, a model of this measurement error can be derived or estimated. This model describes how likely it is to obtain any particular measurement (here the mass that is deduced from the weight measurement and the model equation) when a given set of system parameters (here the true mass) is assumed. This model can be written as the following pdf:

$$
\text { measurement } \mapsto p(\text { measurement } \mid \text { system or model parameters })
$$

Taking the notations introduced earlier, the pdf that interests us is the following one:

$$
\left\{z_{i} \mid i \in \llbracket 1, m \rrbracket\right\} \mapsto p\left(\left\{z_{i} \mid i \in \llbracket 1, m \rrbracket\right\} \mid \theta\right) .
$$

Assuming that the error on each measurement does not depend on the other measurements and that these errors follow a Gaussian distribution (whose respective standard deviations are noted $\sigma_{i}$ later on), it can easily be shown (full derivation can be found in [12]) that the maximum-likelihood problem can be written as:

$$
\widehat{\theta}=\underset{\theta}{\operatorname{argmin}}\left(\theta \mapsto \sum_{i=1}^{m} \frac{\left(z_{i}-y_{i}(\theta)\right)^{2}}{\sigma_{i}^{2}}\right)
$$

For the application to the characterization of gust and turbulence using the previously introduced free-form model, it was also found useful to add two Tikhonov regularization terms $[44,45]$ to the least-squares function, which lead to solve the following regularized optimization problem:

$$
\widehat{\theta}=\underset{\theta}{\operatorname{argmin}}\left(\theta \mapsto \sum_{i=1}^{m} \frac{\left(z_{i}-y_{i}(\theta)\right)^{2}}{\sigma_{i}^{2}}+\gamma_{1}\left\|\Gamma_{1} \theta\right\|^{2}+\gamma_{2}\left\|\Gamma_{2} \theta\right\|^{2}\right) .
$$

The used Tikhonov regularization matrices $\Gamma_{1}$ and $\Gamma_{2}$ are respectively of sizes $(p-1) \times p$ and $(p-2) \times p$ (with $p$ being the number of parameters in 
the vector $\theta$ ) and respectively penalize the first and second derivatives of the reconstructed wind profile. To this end $\Gamma_{1}$ is based on the coefficients $[-1,+1]$ and $\Gamma_{2}$ is based on the coefficients $[-1,+2,-1]$ of the well-known Mexican Hat wavelet/convolution filter (also called Laplacian-of-Gaussian filter) [39] of order two.

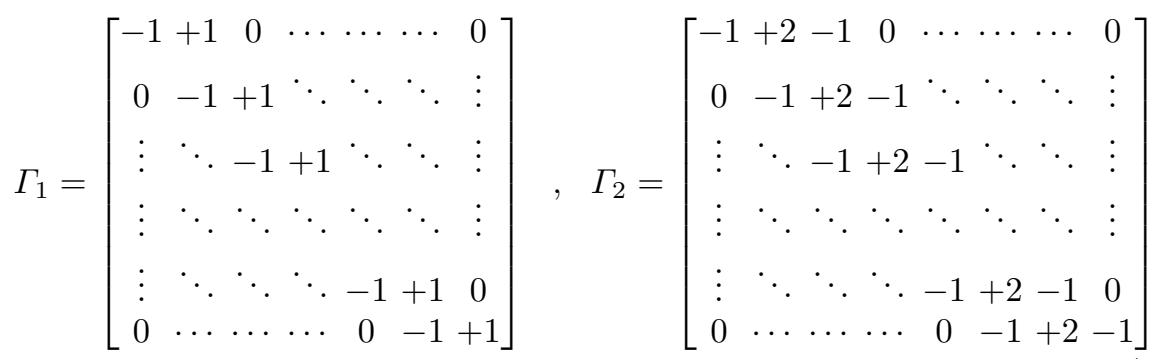

This choice permits to penalize wind fields containing small-scale variations: the small-scale variations are expected to not be well measured by the LIDAR sensor and not be the components of the wind field for which it is beneficial to anticipate and to begin counteracting them very early anyway. The coefficients $\gamma_{1}$ and $\gamma_{2}$ permit to tune the relative strength between the Tikhonov penalization and the least-squares criterion. It was found that even small values of these coefficients (i.e., not massively changing the overall shape of the profile found on the tested cases) were already well penalizing nonsmooth profiles and also helping some of the optimization algorithms to converge more quickly. The regularized problem of Eq. (5) can be solved using many different nonlinear optimization algorithms and the required computation time can be reduced significantly by choosing a well suited method (see section 2.6). However, as long as the problem is well conditioned, most algorithms should be able to find the same global optimum.

\subsection{Results of the Wind Reconstruction Process}

For conciseness reasons, the reconstructed wind profiles are not shown hereafter but can be found in [12]. When a sufficient number of line-of-sight measurements is considered, the overall shapes of the gusts can be reconstructed quite well, but relatively small-scale variations/oscillations around the real wind profile are present. The Tikhonov terms can be used to smooth the reconstructed wind profile but this also leads to a deformation of the overall shape of the reconstructed wind profile. The chosen approach combines a relatively low smoothing and a wavelet-based signal shrinkage in the feedforward controller itself $[8,10]$. This latter process allows to focus on the large-scale variations of the wind profile, which are the ones leading to the peak loads. 
2.6 Solving the Wind Reconstruction Algorithm

\subsubsection{BFGS Algorithm}

The Broyden-Fletcher-Goldfarb-Shanno (BFGS) algorithm is an iterative optimization method to solve nonlinear programming problems. This quasi-Newton method has the advantage of showing relatively good performance for nonlinear problems which are not least-squares problems. During the CleanSky Smart Fixed Wing Aircraft project, it was unclear whether the free-form model structure would be used and whether the wind reconstruction problem would be formulated as a least-squares problem (linear or not). Consequently, using a general purpose algorithm as BFGS was a safe first choice. The actual implementation slightly differs from the original BFGS algorithm. An approximated computation of the descent direction is used (more robust if the problem is not very well conditioned, but almost no influence when it is well conditioned). Once a descent direction has been chosen, an exact line search method switching between different behaviors (e.g. quadratic and cubic approximations) depending on the apparent local shape of the function is used. Towards the end of the work in CleanSky Smart Fixed Wing Aircraft it appeared that the freeform model structure is very well suited for the wind reconstruction and that the least-squares problem (resulting from the maximum-likelihood problem plus Gaussian and independence assumptions) will be kept. For least-squares problems specialized algorithms (e.g. Gauss-Newton) exist that are usually significantly faster than a general purpose algorithm such as BFGS. It should also be noted that this free-form model structure creates very sparse residuals (inside the global cost function). This sparsity has been used in the BFGS algorithm used and is also used in the Gauss-Newton implementation used in this work and presented hereafter.

\subsubsection{Gauss-Newton Algorithm}

The Gauss-Newton (GN) method allows to solve a nonlinear least-squares problem by using the so-called residuals, which are the differences between the real data points (here the measurements) and the values predicted by the regression (here the free-form model evaluated at the same locations and under the same line-of-sight directions). When considering measurements with different levels of noise, each residual should be corrected: for all $i$, the $i$-th residual is multiplied by a factor $1 / \sigma_{i}$, where $\sigma_{i}$ is the standard deviation of the noise distribution of the $i$-th measurements, as shown later in Eq. (9).

If the least-squares problem is linear, the algorithm will find the optimal solution in only one step. Otherwise several iterations might be necessary. This new algorithm has been implemented at the beginning of a follow-up activity as part of the CleanSky 2 Airframe-ITD (Integrated Technology Demonstrator) to improve the real-time properties of the wind reconstruction process. In order to use the GN algorithm, the problem of Eq. (4) is (equivalently) reformulated as: 


$$
\widehat{\theta}=\underset{\theta}{\operatorname{argmin}}\left(\|J \theta-r\|^{2}\right)
$$

and similarly the regularized problem of Eq. (5) can be written in the form:

$$
\widehat{\theta}=\underset{\theta}{\operatorname{argmin}}\left(\|J \theta-r\|^{2}+\|\Gamma \theta\|^{2}\right)
$$

where $\Gamma$ is defined as:

$$
\Gamma=\left[\begin{array}{ll}
\sqrt{\gamma_{1}} & \Gamma_{1} \\
\sqrt{\gamma_{2}} & \Gamma_{2}
\end{array}\right]
$$

The vector $r$ contains the $m$ residuals corresponding to the $m$ measurements considered in the wind reconstruction problem:

$$
\forall i \in \llbracket 1, m \rrbracket, \quad r_{i}\left(\theta^{[k]}\right)=\frac{z_{i}-y_{i}\left(\theta^{[k]}\right)}{\sigma_{i}}
$$

and $J$ is the Jacobian matrix whose coefficients are defined as:

$$
\forall(i, j) \in \llbracket 1, m \rrbracket^{2}, \quad J\left(\theta^{[k]}\right)_{(i, j)}=\frac{\partial}{\partial \theta_{j}} r_{i}\left(\theta^{[k]}\right) .
$$

When applying the Gauss-Newton algorithm to the non-regularized problem of Eq. (4), the parameter vector $\theta$ is updated at each iteration as follows:

$$
\theta^{[k+1]}=\theta^{[k]}+\left(\left[J\left(\theta^{[k]}\right)\right]^{T} J\left(\theta^{[k]}\right)\right)^{-1}\left[J\left(\theta^{[k]}\right)\right]^{T} r\left(\theta^{[k]}\right) .
$$

In the case of the regularized version shown in Eq. (5) the recursion becomes:

$$
\theta^{[k+1]}=\theta^{[k]}+\left(\left[J\left(\theta^{[k]}\right)\right]^{T}\left[J\left(\theta^{[k]}\right)\right]+\Gamma^{T} \Gamma\right)^{-1}\left[J\left(\theta^{[k]}\right)\right]^{T} r\left(\theta^{[k]}\right)
$$

If the matrices $J$ and $\Gamma$ do not depend on the parameters $\theta$ and the residual vector $r$ linearly depends on $\theta$, then the vector $\theta^{[1]}$ (i.e. after the first iteration) will already be the optimum, regardless of the used initial parameter vector $\theta^{[0]}$. The wind reconstruction problem as defined in the previous sections satisfies these assumptions and the Gauss-Newton algorithm finds the optimum in only one step. 
2.6.3 Performance Comparison on Wind Reconstruction Problems of Varying Sizes

Even if the Gauss-Newton is expected to clearly outperform the BFGS algorithm on this problem, a comparison of both algorithms for a given wind reconstruction problem with variable database size was performed with the aim of quantifying the improvements. For this, various optimizations were run on a desktop computer under MATLAB R2007b. Furthermore an evaluation of the respective performances of the 32 bit and 64 bit program versions was done (same computer, but code compiled either for x86 or x64 architectures). During the implementation of the Gauss-Newton algorithm, a few improvements of the BFGS algorithm were identified and performed, which lead to consider both BFGS versions: the original version as used in $[12,10]$ for reference and the slightly modified version to ensure a fair comparison. Both algorithms are implemented in a sparse way: only the required terms are computed which is done by exploiting the natural structure of the problem and of the free-form wind model but without needing to rely on a general purpose sparse linear algebra library for it. On the wind reconstruction problem with the parameter values used about a factor 10 to 15 is gained by exploiting sparsity and this for both algorithms.

The results are shown in Figure 5. In this figure, the computation time required to converge is represented in ordinate and the wind reconstruction problem size in abscissa. The problem sizes were varied by changing the Doppler LIDAR sensor parameters: some of the LIDAR configurations used might be not representative of any feasible LIDAR setup but provide a representative wind reconstruction problem for the assessment of the computation time needed by both algorithms.

The 64 bit architecture provides a final optimization result with lower computation time than 32 bit (as expected), but the relative results are well comparable with regard to the performance of each algorithm with an identical database size. The slope of the average computation time as function of the database size is significantly smaller for the Gauss-Newton (GN) algorithm because the wind reconstruction problem can be solved in only one iteration whereas the BFGS needs several iterations to converge. Therefore the GN is preferred for future work because it is able to provide a higher update rate of the wind profile even when considering a large number of measurements. This was desired to enable the use of the wind reconstruction algorithm

- with a higher update rate $(10 \mathrm{~Hz}$ seems a good target for most airplanes),

- with LIDAR configurations with higher spatial resolution (and thereby producing more measurements) even if each measurement is affected by a larger error.

The experience gained in the CleanSky Smart Fixed Wing Aircraft project indicates that these are the two directions (higher update rate and higher spatial resolution) which would improve the potential for feedforward load alleviation based on Doppler LIDAR sensors. 


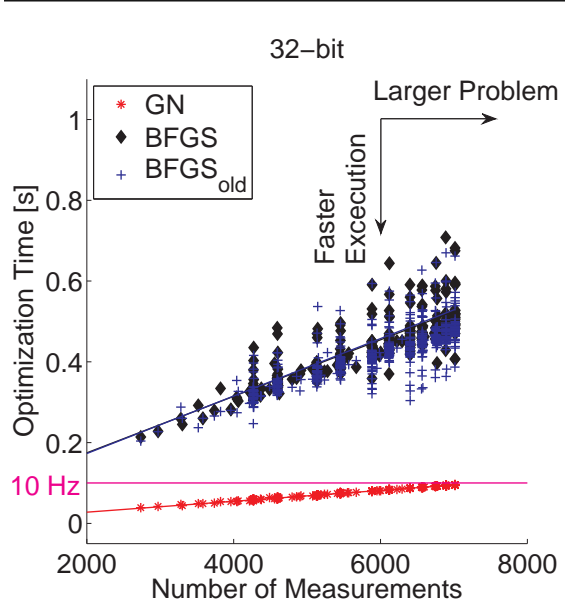

(a) 32 bit Matlab R2007b

BFGS $7.3964 \cdot 10^{-5}$ s per measurement

GN $\quad 1.3589 \cdot 10^{-5} \mathrm{~s}$ per measurement 64-bit

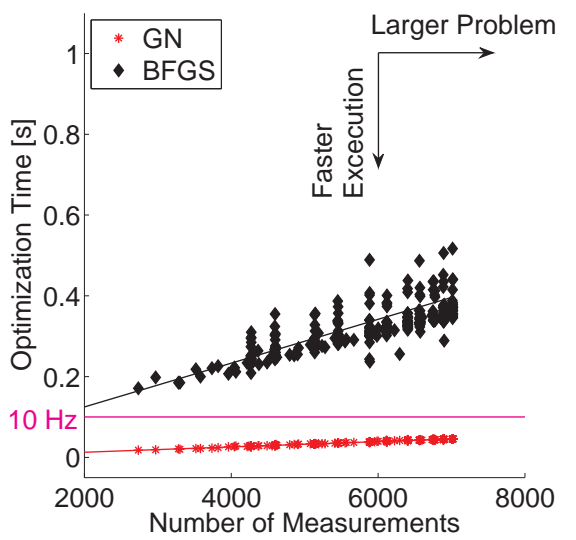

(b) 64 bit Matlab R2007b

BFGS $5.3862 \cdot 10^{-5}$ s per measurement

GN $\quad 0.6528 \cdot 10^{-5} \mathrm{~s}$ per measurement

Fig. 5: Comparison of optimization run time consumption of GN and BFGS algorithm for solving a given wind reconstruction problem with measurement databases of increasing sizes

\subsection{Feedforward Load Alleviation Controller}

In the specification of the desired load alleviation behavior made by the load specialists, a typical wording came repeatedly: "small-amplitude disturbances should not be alleviated using the spoilers." While this sounds and definitely is reasonable from an airplane performance point of view, this implicitly specifies that a highly nonlinear allocation constraint is desired for the controller. This constraint makes the direct application of some of the most powerful tools and results in control theory impossible. It also raised the question of defining a simple controller structure, which can easily be tuned and permits to obtain such a highly nonlinear behavior. Pitching the aircraft up or down is the most effective way to change the aerodynamic loads. The aerodynamic loads can also be modified through control surfaces (e.g. ailerons and spoilers on the wing) or even direct-lift control (DLC). Spoilers can be used to reduce loads but not to increase them, at least not in the steady case or with regular deflection speed [13]. The use of spoilers for ride control is therefore more restricted than DLC, even if spoiler deflections with the correct timing can improve the ride quality. DLC and spoilers are less effective than a change of angle-of-attack in terms of lift change, however their effect on drag and thereby on the longitudinal accelerations is relatively high in comparison. These longitudinal accelerations are very uncomfortable and must be compensated: a compensation function was already in the LARS system, but with the typical control surface designs this compensation leads to more drag on average (even in calm air), such that 
this compensation function shall ideally be activated separately when flying in turbulence, as proposed in [16].

For an effective load alleviation based on pitching actions and with a restricted bandwidth, the pitching commands shall be initiated before encountering the disturbance. After having considered the motivations for such a desired alleviation behavior, the practical aspects regarding structural loads at the HTP and the fuselage as well as for passenger comfort, the proposed alleviation concept was finally expressed as follows:

- The low frequencies of the atmospheric disturbances should be alleviated by pitching the airplane up or down. For passenger comfort reasons, it shall be possible to select a different behavior for small-amplitude disturbances or even to restrict this behavior to large-amplitude disturbances (i.e. relevant for peak loads).

- In the medium-frequency range:

- The tuning parameters should allow to choose whether disturbances with very small amplitudes in the medium-frequency range are alleviated or not (e.g. to avoid unnecessary actuator cycles or to reduce power consumption).

- The disturbances with relatively small amplitudes should be alleviated using only trailing edge deflections or camber variations (i.e. basically with ailerons but possibly also with innovative flaps if available).

- The larger disturbances should be alleviated with any possible means, including spoilers or any other suitable control device even if they tend to deteriorate the airplane's aerodynamic performance.

- The higher-frequency components of the disturbance will not be alleviated at all with the feedforward function.

The limits between "low," "medium," and "higher" frequencies as well as the thresholds between "very small," "small," and "larger" amplitudes are tuning parameters for the feedforward load alleviation function. Note that the bandwidth of the feedback load alleviation function (section 3) might be tuned to be higher than the one of the feedforward function. There is a priori no reason to impose any relationship between the bandwidths of the feedforward and feedback gust alleviation functions. However, the achievable wind sensor spatial resolution will probably limit the effective bandwidth of the feedforward gust alleviation function. Note that the definition of the allocation strategy is strongly dependent on the considered aircraft and on the critical gust lengths that the feedforward should target primarily. The analysis of the needs of the XRF-1 configuration (flexible long-range aircraft with the critical gust length between 300 and $350 \mathrm{ft}$ ) led to this particular decomposition, but a different decomposition might be preferable for other aircraft, for instance for a more rigid business jet aircraft with the critical gust length between 80 and $150 \mathrm{ft}$.

This concept was successfully implemented using the so-called Fast Orthogonal Wavelet Transform (FOWT), which is one of many time-frequency/scale techniques that could be used in order to obtain the aforementioned behav- 
ior. The reader is referred to [8] for the implementation details and a detailed discussion on the structure of this feedforward controller.

\section{Optimization-Based Multi-Objective Robust Control Law Synthesis}

\subsection{Overview of the Optimization-Based Multi-Objective Robust Control} Law Synthesis Methodology

Flight control law design is a multi-variable control problem where various strict requirements have to be satisfied. In case of uncertainties, missing or erroneous feedback or scheduling variables robustness of the controller is indispensable. To tackle these problems, an optimization-based multi-objective synthesis approach is proposed $[26,27,36,35]$. The main features of this methodology are

1. that various kinds of design objectives can be taken into account in their most natural form (e.g. initial response, overshoot, loads, comfort),

2. that design alternatives can be assessed most visibly with respect to given requirements,

3. that robustness can be considered in various ways (e.g. multi-model approach, robustness criteria).

In case of various, usually conflicting, design objectives the designers need to be able to compare different designs and they need to know up to which extent a design objective is achieved. In case of conflicts they need quantitative information about degradation in individual objectives while other objectives are improved. Such performance indices or criteria should accurately reflect the design objectives and provide a comprehensive measure of the achieved design quality. Mathematical formulation of design objectives as criteria also allows the computer to distinguish different designs. Multi-objective optimization, as a computer-aided design technique, is able to take care of all the various conflicting design goals individually, but compromising them concurrently. In case of available quantitative information about requirements and demands the problem can be solved by transforming the set of criteria into a weighted min-max optimization problem, where the weights are chosen according to the demands. On the other hand to explore system performance in the criteria space no quantitative information about requirements and demands is necessary. In that case the problem must be solved as a vector optimization problem leading to a Pareto-optimal solution set.

The whole process of robust control law synthesis based on multi-objective optimization is performed in several steps. At each step of this process, one or more optimization problems are solved numerically. The results of these optimizations are analyzed by the designer. In multi-objective optimization there usually exists no unique "optimal" solution, but a (usually infinite) set of Pareto-optimal solutions, called compromise solutions, for which an improvement in one design objective may cause degradation in one or more of the 
other objectives. The designer tries to find the best overall compromise given the various, usually conflicting objectives and constraints. As long as the designer is not fully satisfied with the current solution, new options (e.g. change of controller structure) might be considered and lead to start a new design step. In order to ease the use of numerical optimization techniques, quantitative and normalized representations of the various objectives are required (see section 3.1.3). This normalization of the objectives and constraints is also very helpful for the detection of possible deficiencies over the range of parameter uncertainty. Model uncertainties should always be considered in model-based design, as no model can be assumed to be perfect. Note that optimization techniques can also be used to find "worst-case" parameter combinations to decide whether a design is robust or not.

\subsubsection{Acceptable Controller Structures and Parameterizations}

Compared to many other control design techniques, directly optimizing the controller parameters - as it is done in the proposed multi-objective design method - permits to avoid restrictions in terms of structure and properties (e.g. linearity) for controller and model. The controller structure can be chosen in a problem-adequate way taking advantage of the designer's knowledge and prior expertise. In classical controller structures the parameters to be tuned are the gains and filter constants. In (linear) control theory there exist several analytic controller synthesis methods, which guarantee structural stability properties. Such methods are for example LQR-synthesis, eigenstructure assignment or $\mu$-synthesis. In any synthesis method there are some free parameters to be determined by the designer in order to define the controller completely. Since the controller structure is arbitrary in multi-objective design, it is possible to incorporate synthesis formulae (like $\mu$-synthesis) into the computational procedure. Hence structural properties of the controller are guaranteed by the synthesis formulae while the synthesis parameters are properly tuned according to the design criteria by multi-objective optimization. The controller structure for the application to the XRF-1-based benchmark model is described in section 3.2.2. It involves some nonlinearities (deadzones and saturations) and exploits the fact that the multi-objective control design technique presented here can deal with nonlinear controllers.

\subsubsection{System Model Description and Robustness}

In principle, there is no particular restriction on the model structure, the types of disturbance to consider, or the simulation tool (linear, nonlinear, multibody simulation software, etc.), as long as they permit to evaluate/simulate the entire system for the design cases and to recover the corresponding results. Robustness of the controller to be designed can be achieved in several ways by appropriate mapping of the robustness requirements onto the design criteria.

1. "Local" robustness criteria 
Robustness of the controller "around" a design point can be enforced in the multi-objective approach by adding suited robustness criteria (e.g. gain/phase margins) to the set of performance criteria.

2. "Global" robustness using the multi-model approach

Robustness against structured parameter deviations is achieved by applying a common controller to a set of fixed "worst-case parameter" system models. This model set characterizes the range of dynamics variations within the range of operation. For each of these models the appropriate list of criteria has to be specified. By combining all criteria together, the problem to design a common controller for several system models simultaneously is transformed to a so-called multi-model multi-criteria design problem. In general there exists no theory that guarantees stability or performance robustness across the range of operation, if only a finite number of operating points is considered. If deficiencies exist in some points, the according operating point has to be added to the multi-model set and a re-design has to be done.

3. Robustness via risk computation using Monte Carlo simulation

Fast simulation code gives the possibility to use Monte Carlo-based risk computation within the synthesis loop. In a Monte Carlo simulation not only a few "worst-case parameter" system models are considered simultaneously, but a lot of simulations are performed with randomly disturbed parameters. However, the performance criteria are not treated separately for each model but are combined to statistical characteristics like mean, standard deviation or risk probabilities which serve as robustness objectives. This means that the requirements stated in JAR-AWO 131 or FAR 25 as well as many others can be used as synthesis criteria directly.

\subsubsection{Criteria Formulation}

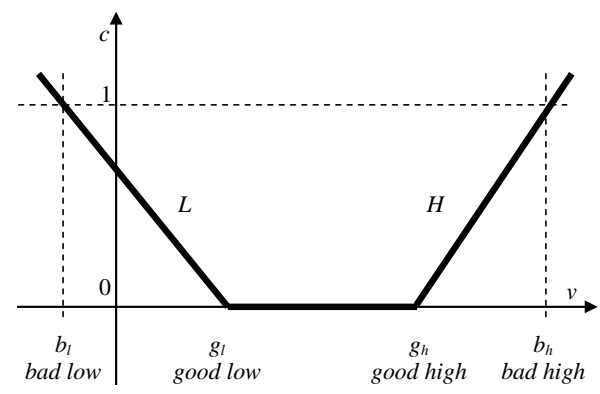

Fig. 6: Transformation of characteristic quantities to optimization criteria.

In engineering design there exist a lot of characteristic quantities to judge design results. But these quantities do not necessarily have the required property of a mathematical criterion needed for multi-objective design. Without 
loss of generality we can assume that an optimization criterion has to be positive real and is to be minimized. Any arbitrarily defined scalar characteristic quantity $\nu$ can be transformed into a compliant criterion form $c$ by the following transformation:

$$
\begin{aligned}
c & =\max (L(\nu), 0, H(\nu)) \\
L(\nu) & =\left(\nu-g_{l}\right) /\left(b_{l}-g_{l}\right), \text { with } b_{l}<g_{l} \\
H(\nu) & =\left(\nu-g_{h}\right) /\left(b_{h}-g_{h}\right), \text { with } g_{l}<g_{h}<b_{h}
\end{aligned}
$$

Each characteristic quantity $\nu$ must be transformed like this individually if necessary. The transformation described in Eq. (13) and illustrated in figure 6 maps any quantity to a non-negative real number and the transformation parameters $b_{l}, g_{l}, g_{h}, b_{h}$ have to be chosen according to the following fuzzy-like description of the objective goal in terms of "bad" and "good":

- A characteristic quantity $\nu$ is considered to be satisfactory or good for values between $g_{l}$ and $g_{h}$.

- It is considered as not acceptable or bad for values less than $b_{l}$ or greater than $b_{h}$.

By such a transformation satisfactory characteristic quantities are mapped to zero (or almost zero due to the approximation of the max-function). This means that a satisfactory characteristic quantity makes no contribution to the overall objective function. However, if a characteristic quantity becomes unsatisfactory, the criterion value increases and contributes to the overall objective again and a traded-off with other objectives might be needed if a conflict arises.

\subsubsection{Assessment and Wort-Case Search for Increased Robustness}

Any designed control law has to be assessed whether the requirements are satisfied over the whole flight envelope for any possible combination of uncertain parameters. The problem is how to detect deficiencies. Assume that the uncertain and operational parameters are bounded and combined in a vector $P$. Of course, any criterion or constraint $c_{i}(P, T)$ is also a function of $P$. Deficiency detection can now be formulated as a global optimization problem

$$
\max _{P} \max _{i}\left(c_{i}(P, T) / d_{i}\right) \quad \text {, subject to } \quad c_{j}(P, T) \leq d_{j}
$$

with the same criteria or constraints as defined for design [28]. A deficiency occurs if the optimization results in a value greater than one. In order to be sure to detect the overall maximum, global optimization procedures are necessary but induce large computational costs. However, experience showed that in the case of insufficiently robust solutions, 'local' optimization procedures are usually able to find local bad/deficient solutions very rapidly, which is sufficient for the next design step. The robustness can be improved by adding the bad cases found in the considered multi-model set and by restarting the optimization for this augmented problem. When dealing with parameter-varying systems, the analysis of the bad cases can also help to detect possibly missing scheduling parameters for the controller. 
3.2 Design of a Feedback Control Alleviation System for the Benchmark Model

\subsubsection{The Benchmark Model}

The design of the feedback active load controller (FBALC) is based on the project's benchmark model described in [37]. From the delivered flight cases it was possible to consider a cruise flight scenario with a speed of about $175 \mathrm{~m} / \mathrm{s}$ described by the data of the two flight cases $\mathrm{Ma}=0.86$ at Height $=8279 \mathrm{~m}$ and $\mathrm{Ma}=0.5$ at Height $=0 \mathrm{~m}$.

The FBALC to be developed should be robust against load variations. These variations are covered in the benchmark model by the 7 load cases F000, FA2M, FA2T, FA9M, FA9T, FC8T and FT8T. Gust disturbances are modeled as discrete 1-cosine gusts with different gust lengths of 30, 150, 300 and $350 \mathrm{ft}$. Only vertical gusts are considered.

\subsubsection{Feedback Control Law Structure}

The principle structure of the FBALC is a simple feedback of the vertical acceleration signal $a_{z, \text { sensor }}$ (from the inertial reference systems) on symmetric ailerons, spoilers and elevators. Only longitudinal motion is considered. Each surface has its own loop consisting of gain, saturation limits and a low-pass filter to suppress high-frequency excitations. Thresholds (noted $l_{\text {elevators }}, l_{\text {ailerons }}$, and $l_{\text {spoilers }}$ hereafter) are introduced to avoid activity of the FBALC already for small accelerations. Inner and outer ailerons and the elevator use the same threshold. The threshold for the spoiler feedback signal is higher than the other. Hence spoilers are activated only for heavy gusts. Vertical acceleration pilot commands are filtered with a third-order linear filter scheduled by Mach number and mass:

$$
\begin{aligned}
\forall i \in \llbracket 0,3 \rrbracket, C_{i}=C_{i, 0} & +\left(M a-M a_{\mathrm{ref}}\right) C_{i, M a}+\left(M-M_{\mathrm{ref}}\right) C_{i, M} \\
\dot{x} & =\left[\begin{array}{ccc}
0 & 1 & 0 \\
0 & 0 & 1 \\
-C_{1} & -C_{2} & -C_{3}
\end{array}\right] x+\left[\begin{array}{l}
0 \\
0 \\
1
\end{array}\right] \delta_{\text {pitch }} \\
a_{z, \mathrm{cmd}} & =\left[\begin{array}{ccc}
C_{0} & 0 & 0
\end{array}\right] x
\end{aligned}
$$

The third-order filter of Eqs. (16-17) is written under the classical horizontal companion form. In this state realization the first element of the state vector $x$ is proportional to $a_{z, \mathrm{cmd}}$, with factor $C_{0}$ between the two as indicated in Eq. (17). The second and third elements of the state vector $x$ are simply the first and second time derivatives of the first state, see two first lines of the state matrix in Eq. (16). The behavior of this filter can be tuned through the coefficients $C_{0}, C_{1}, C_{2}$, and $C_{3}$ and usually consist in a third-order 
low-pass behavior. The commanded vertical acceleration is compared to the measured acceleration and used in combination with a threshold function $T$, a proportional controller $K_{i}$, and a cut-off filter $F(s)$, as shown in Eqs. (18-20).

$$
n_{z, \text { error }}=\left(a_{z, \mathrm{cmd}}-a_{z, \text { sensor }}\right) / g
$$

$\forall i \in\{$ elevators, ailerons, spoilers $\}, \quad \delta_{i}=F(s) \quad K_{i} T\left(n_{z, \text { error }}, l_{i}\right)$

$\forall(x, y) \in \mathbb{R} \times \mathbb{R}^{+}$, if $|x| \geq|y|, T(x, y)=x$, otherwise $T(x, y)=0$

\subsubsection{Design Goals and Applied Loads and Comfort Criteria}

The overall design goals for the FBALC are:

- Robust gust load alleviation at wing root.

- Compliance with design loads at all other load stations under consideration. Since design load values are not available in this benchmark the design goal will be to keep an increase of loads at other stations than the wing root as small as possible.

- Do not degrade passenger comfort, improve if possible.

- No effect on handling qualities.

The loads criteria considered are the RMS/Max/Range-value of shear force $F_{z}$, bending moment $M_{x}$ and torsion $M_{y}$ as response to a 1 -cosine gust at the stations modeled for the benchmark aircraft.

Passenger comfort is measured as a global comfort criterion for seated persons according to the ISO 2631-1 standard [34]. It is a frequency-weighted criterion based on IRS vertical acceleration $a_{z \text {,sensor }}$.

The effect of the FBALC system on handling qualities and maneuverability is not explicitly considered during the design.

\subsubsection{Design Setup}

The design is performed applying the optimization-based multi-objective robust control law synthesis approach described in section 3.1. Robustness of the control law is incorporated by the multi-case approach outlined in section 3.1.2. The design scenario under consideration and the available data allow for 56 different cases covering flight conditions, mass, and gust variations. These cases are combinations of the seven load cases available, the two Mach/altitude combinations as well as the four different gust lengths. To reduce the computational burden 8 cases have been selected representing the most critical cases regarding the design goals. These cases are the combinations of load case FA2T with Mach 0.5 and Mach 0.86 and the four gust lengths of $30,150,300$ and $350 \mathrm{ft}$. 
For each cut station the RMS, maximum and range values of the shear force $F_{z}$, the bending moment $M_{x}$ and the torsion moment $M_{y}$ are computed. Only the right cut stations are considered since the flight scenario is symmetric. For each case this results in 276 loads criteria which have to be taken into account during optimization. The six criteria concerning the loads at the wing root have to be minimized, the remaining 270 loads criteria are treated as constraints with prescribed upper bounds reflecting the limit loads for each station.

The proposed control law structure has seven parameters to be tuned: four gains of the feedback loops for inner/outer aileron, elevator and spoilers; two threshold parameters and one cut-off frequency of the identical low-pass filters to avoid high-frequency excitations.

\section{Results for the XRF-1 Benchmark Model}

\subsection{Performance of the Feedback Active Load Controller (FBALC)}

\subsubsection{Load Alleviation}

Several multi-objective optimization runs have been performed to achieve the following results. The runs have mostly been necessary because of the lack of information about the design loads (see second point of section 3.2.3). Hence several evaluations had to be done in order to properly define the upper bounds for the constraints representing the unknown design loads. Normally these design loads would be known and this step would then be unnecessary.

The quantitative result is (partly) depicted in figure 7. It shows the achieved criteria values in parallel coordinates for four cases representing different gust lengths $(30,150,300$, and $350 \mathrm{ft}$, from top to bottom). The color of the ordinate axes (green, red, blue) indicates the usage of the criterion for minimization (green), as constraint (red) or for observation only (blue). Each criteria is normalized with the methodology presented in section 3.1.3. Note that some criterion are repeated twice: once as objective function and once as constraint. The achieved numerical values prior to normalization are the same, but the normalization factors are different. A level of one for the constraint represents the maximum acceptable value (hard limit), whereas the same level of one for the objective function represents the desired performance (target, corresponding to lower non-normalized value than for the constrained value equal to one).

In figure 7 , the normalized values obtained for all criteria are connected by a line. The dashed light magenta line shows the values for a gust response without FBALC, whereas the dark blue line corresponds to the same cases with FBALC. It can be seen that loads can be reduced at many stations for almost all gust lengths considered (blue below red). Only for the shortest gust with a length of $30 \mathrm{ft}$ (top most plot) almost no improvement could be obtained, which is not problematic since the loads are small in this case. Sig- 

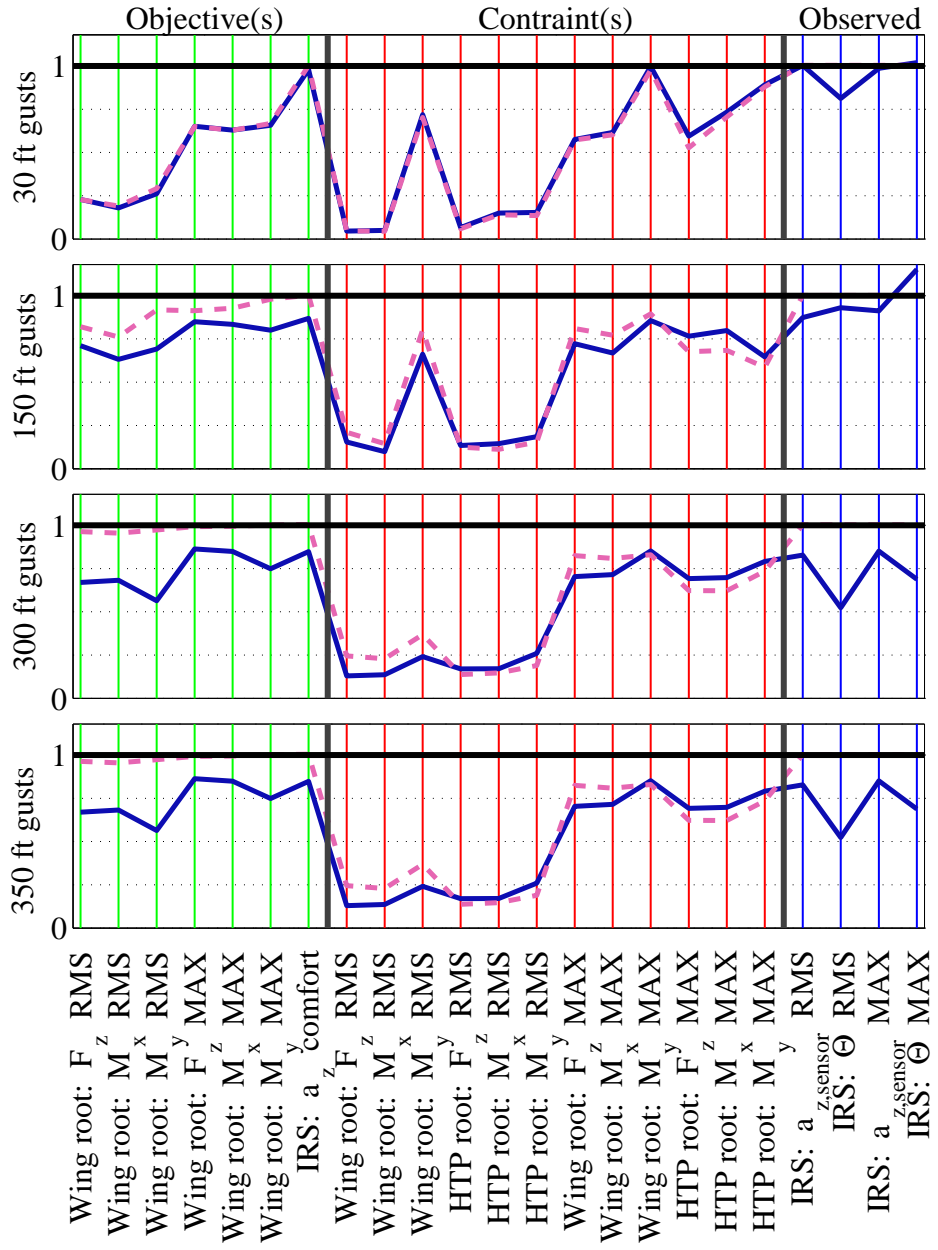

Fig. 7: Criteria representation in parallel coordinates, cases with different gust lengths (dashed light magenta: FBALC off, dark blue: FBALC on). Normalized values: all criteria are normalized individually prior to optimization.

nificant improvements are obtained on the objective functions (seven criteria on the left) for the gusts with lengths 150, 300, and $350 \mathrm{ft}$. As the constraints were already satisfied, no such trend is observed on the contraints: some are slightly improved, others are slightly deteriorated, but most importantly none of them violates the hard constraints imposed. Finally, the observed criteria (i.e. computed and saved during the optimization but not directly optimized) also tend to be improved. For these criteria, the aircraft without FBALC was taken as reference, which explains why its performance is equal to one for all observed criteria. The only exception is the maximum pitch angle $\Theta$ obtained 
in the $150 \mathrm{ft}$ case, which was increased by about $15 \%$. Whilst this remain not critical, this gives an indication that the pitching motion of the aircraft was slightly increased by the FBALC in that case. Please note that for conciseness reasons only a small portion of the optimization criteria used in the FBALC controller design and presented in section 3.2.3 could be represented for all 56 different cases (flight conditions, mass, and gust variations) in figure 7 . The "parallel coordinates"-based graphical representation used in this figure is a very useful during control design as it gives a very good overview of the obtained performance across all the cases and criteria in a very compact representation.

In order to get even more physical insight into the loads envelopes along the wing and the horizontal tailplane (HTP) are shown hereafter. 35 stations were defined along each wing, and 11 stations were defined along each side of the HTP. For each of these stations and for each of the 56 available cases (flight point, mass, gust, etc.) the loads time series (as result of a simulation in the time domain) were evaluated based on three metrics: RMS, range (max-min), and peak value. Each of the 168 results $(56 * 3=168)$ obtained for each station can be analyzed separately but global performance indexes are also very useful to avoid being overwhelmed by the amount of performance indexes. The average and maximum loads in the 56 cases are considered and combined with the three metrics (RMS, range, peak). This leads to six performance indexes per load station and per loads type. Note that in the following, the term "maximum" always refers to a maximum over the 56 different cases, whereas the term "peak" denotes the maximum value reached during a time simulation for one particular case. As a consequence the "maximum peak value" denotes then the maximum value for all 56 cases of the peak values, each of which being the maximum value over time for one case. The three considered loads types are the shear force $F_{z}$, the bending moment $M_{x}$, and the torsion moment $M_{y}$. One of the most critical locations in order to enable weight savings is the wing root. The 18 performance indexes ( 6 indexes and 3 loads types) for the wing root are shown in table 1 . The multiplicity of the indexes is linked to the fact that they physically relate to various interesting quantities. For instance, the mean of all cases in terms of the RMS and of the range will be rather interesting for fatigue, whereas the maximum peak values in all cases will rather be interesting for the required overall strength of the structure.

All the aforementioned criteria were taken into account for all wing and HTP stations during the present work. For conciseness reasons, only the maximum values (over all 56 cases) of the RMS and the peak loads are shown in figures 8 and 9 . These figures show that the shear force $F_{z}$ and bending moment $M_{x}$ loads are reduced both in RMS and peak value along very large portions of the wing (top three plots). Only torsion $M_{y}$ is slightly increased at some of the stations 3 to 35 of the wing. A consequence of the active load alleviation controller is that the maximum RMS values of shear force $F_{z}$ and bending moment $M_{x}$ are increased for the HTP (two top right plots in figure 8). These increased values are however still below the prescribed level for 
design and were consequently accepted. All other measures (including those not shown here) indicate an overall decrease of HTP loads.

Table 1: Load reductions at wing root obtained with the FBALC

\begin{tabular}{|c|c|c|c|c|c|c|}
\cline { 2 - 7 } \multicolumn{1}{c|}{} & \multicolumn{3}{c|}{ Mean } & \multicolumn{3}{c|}{ Maximum } \\
\cline { 2 - 7 } \multicolumn{1}{c|}{} & RMS & Range & Peak & RMS & Range & Peak \\
\hline Shear force $F_{z}$ & $10 \%$ & $10 \%$ & $6 \%$ & $12 \%$ & $1 \%$ & $1 \%$ \\
\hline Bending moment $M_{x}$ & $17 \%$ & $15 \%$ & $6 \%$ & $17 \%$ & $12 \%$ & $9 \%$ \\
\hline Torsion moment $M_{y}$ & $26 \%$ & $22 \%$ & $9 \%$ & $20 \%$ & $20 \%$ & $10 \%$ \\
\hline
\end{tabular}

\subsubsection{Behavior During Pitch Maneuvers}

The effect of the pre-filter to compensate pilot input and thereby to decouple active loads control from maneuver-induced vertical accelerations is demonstrated in figure 10. A $1 \mathrm{~g}$ maneuver was simulated for load case FA9M at Mach 0.5. The gust applied had a length of $150 \mathrm{ft}$ and, when present, started at $t=2.5 \mathrm{~s}$. From figure 10 it can be seen that - as desired - vertical acceleration as well as the control surface deflections are not affected by the FBALC controller during the maneuver in the absence of any gust: the green line (no gust, FBALC on) lies exactly on the black line (no gust, no FBALC). However, when a gust was applied the FBALC controller reduces accelerations also during the maneuver. The lower three diagrams of figure 10 show the control effort necessary for gust load alleviation. The additional elevator deflection is quite small, whereas the symmetrical deflections of the ailerons and of the spoilers are up to $5^{\circ}$. Though not shown here (results can be found in [9]) the gust loads at the wings and HTP are reduced by the designed FBALC controller when active and in the presence of the gust (compared to the case without active load alleviation and in the presence of the gust).

\subsubsection{Impact on Comfort}

The structural modes of large flexible aircraft tend to be of lower frequencies (than those of small and more rigid aircraft) and to negatively impact the passenger comfort. In [34] comfort criteria based on the ISO 2631-1 standard [1] are defined. They comprise "low frequency comfort" determined by vibrations but also motion sickness phenomena caused by very low frequencies. Those criteria are very well suited for the multi-objective integrated design of flight control laws and gust load alleviation functions.

In this application comfort improvement was not of primary interest. The goal was to not decrease comfort while gust loads are alleviated. The comfort criterion was therefore used as a constraint with upper bounds corresponding to the values of the FCS-augmented aircraft: the basic controller serves as baseline and the FBALC is not allowed to deteriorate the comfort values compared to this baseline. In figure 11 three bar charts are depicted showing 

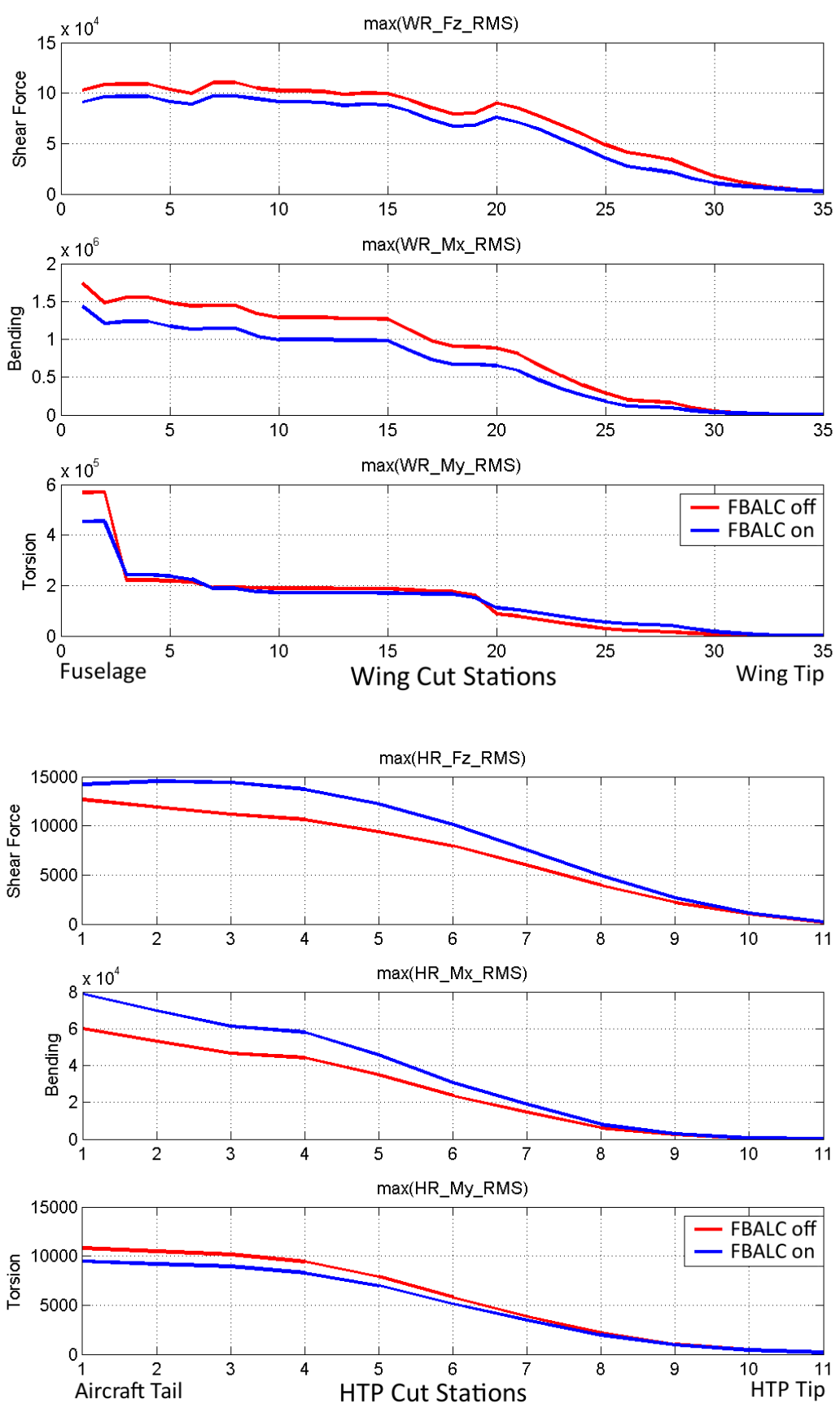

Fig. 8: Maximum RMS values of loads $F_{z}, M_{x}$, and $M_{y}$ along the right wing (top plots, 35 cut stations) and the right HTP (bottom plots, 11 cut stations). 

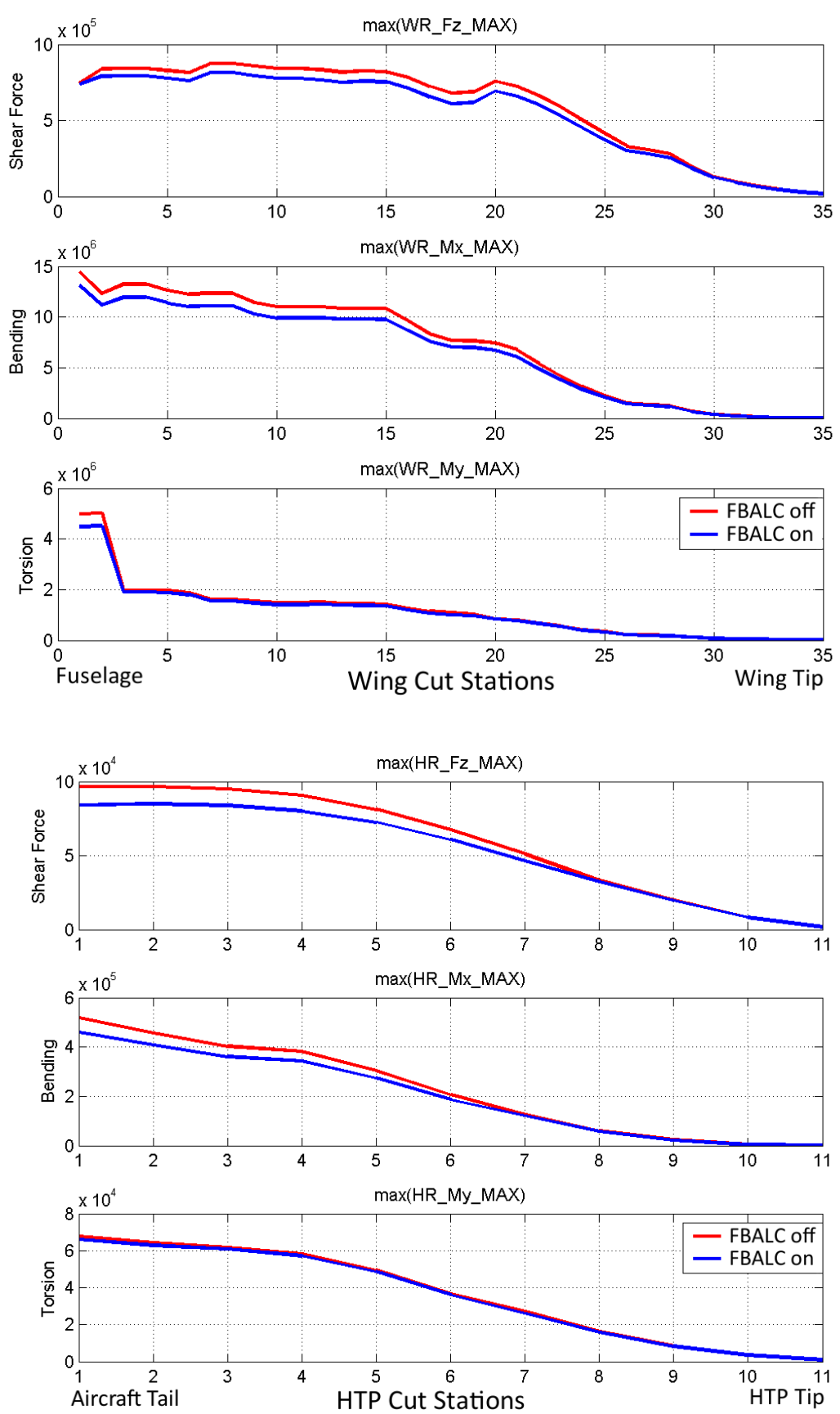

Fig. 9: Maximum peak values of loads $F_{z}, M_{x}$, and $M_{y}$ along the right wing (top plots, 35 cut stations) and the right HTP (bottom plots, 11 cut stations). 

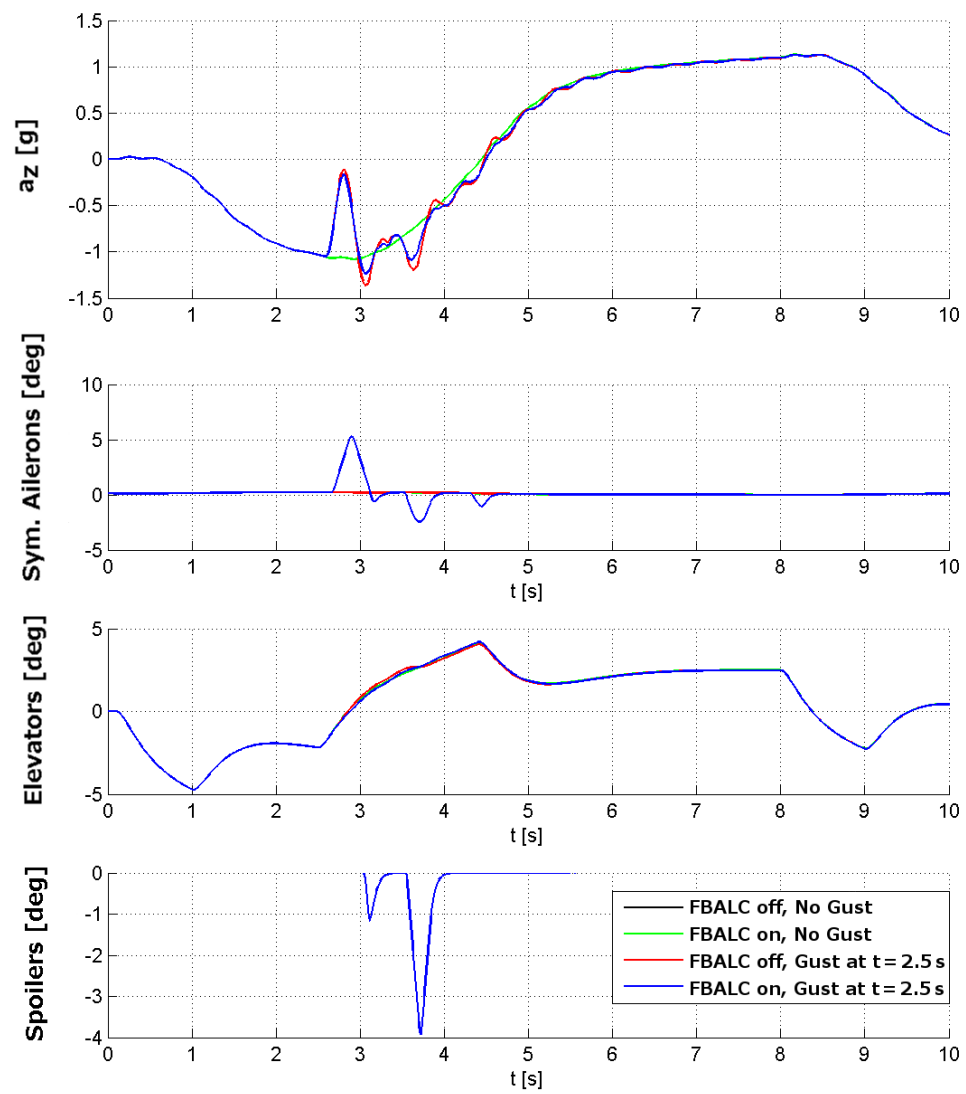

Fig. 10: Response of the vertical acceleration $a_{z}$ and control surfaces during a $1 \mathrm{~g}$ maneuver: with/without gust and with/without active load alleviation. (Mach 0.5, load case FA9M, gust length $150 \mathrm{ft}$ ).

the comfort indexes achieved for a 1-cosine gust of lengths $30 \mathrm{ft}, 150 \mathrm{ft}$ and $350 \mathrm{ft}$ respectively. The 14 cases correspond to the possible combinations of Mach number (2) and load cases (7). It can be seen that comfort is positively affected by the FBALC system for longer gusts. For short gusts the comfort index remains unchanged and does not exceed the design demands, as required.

4.2 Performance of the Integrated Feedback and Feedforward Load Alleviation Functions

A simulation model that couples both the feedback load alleviation function (FBALC) that was presented in section 3 and the feedforward load alleviation function ("Gust Load Alleviation using REmote WInd SEnsors and TimeFrequency-based Allocation Constraints" or GLAREWISE+TFAC) that was 

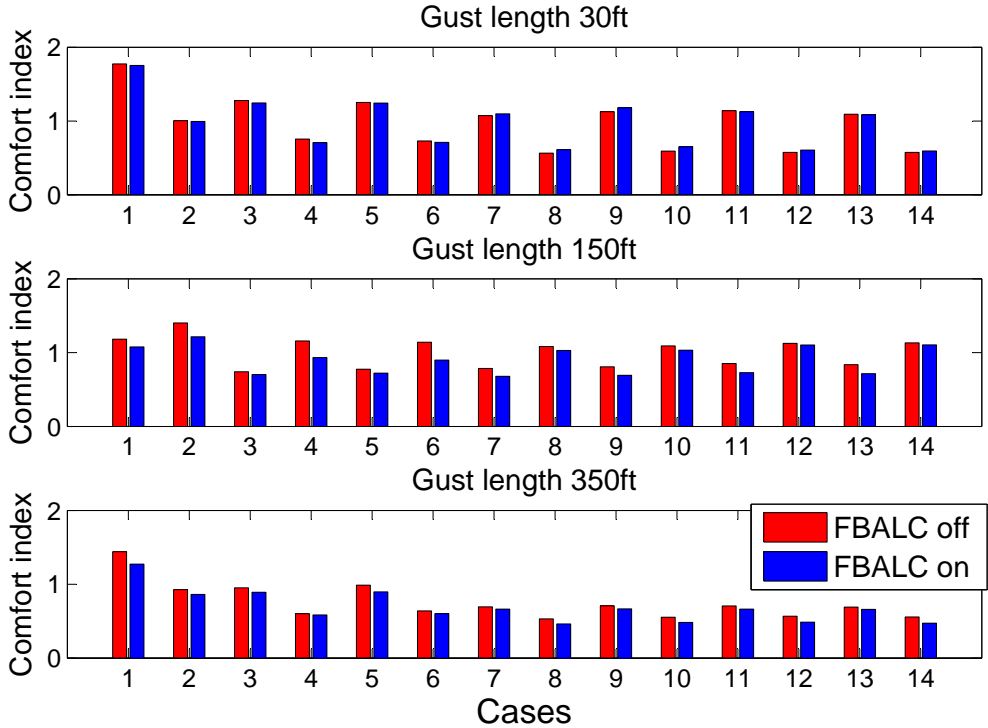

Fig. 11: Comfort index for 3 different gust lengths and the 14 possible combinations of Mach and load cases.

presented in section 2 was developed. It was used to make a first evaluation of the load alleviation performance improvement achieved by the feedforward load alleviation in comparison to the load alleviation performance of the feedback load alleviation alone.

Three cases will be exemplarily shown hereafter for various gust encounters:

1. Only basis flight control system or EFCS (typical Airbus-like $n_{z}$-law in the pitch axis and rate-command/attitude-hold in the roll axis)

2. EFCS and FBALC

3. EFCS, FBALC, and GLAREWISE+TFAC.

The GLAREWISE + TFAC function is not meant to be used without feedback load alleviation. The combination EFCS and GLAREWISE+TFAC is therefore not considered hereafter. The considered gusts have a one-minuscosine $(1-$ cosine $)$ shape and all have the same amplitude here. Their lengths are: $30 \mathrm{ft}, 150 \mathrm{ft}, 300 \mathrm{ft}$, and $350 \mathrm{ft}$. They were considered in both directions: upward and downward.

Figure 12 shows the results of the simulations for the $350 \mathrm{ft}$ and $300 \mathrm{ft}$ gust lengths and figure 13 shows the results of the simulations for the $150 \mathrm{ft}$ and $30 \mathrm{ft}$ gust lengths. On both figures and for each simulation the wing root bending moment is shown on the left and the vertical load factor $n_{z}$ in the middle of the cabin on the right. The black line corresponds to the "EFCS" case, the dashed magenta line corresponds to the "EFCS and FBALC" case, and finally the dash-dotted cyan line corresponds to the "EFCS, FBALC, and GLAREWISE + TFAC" case. The anticipation capability of the GLAREWISE+TFAC 

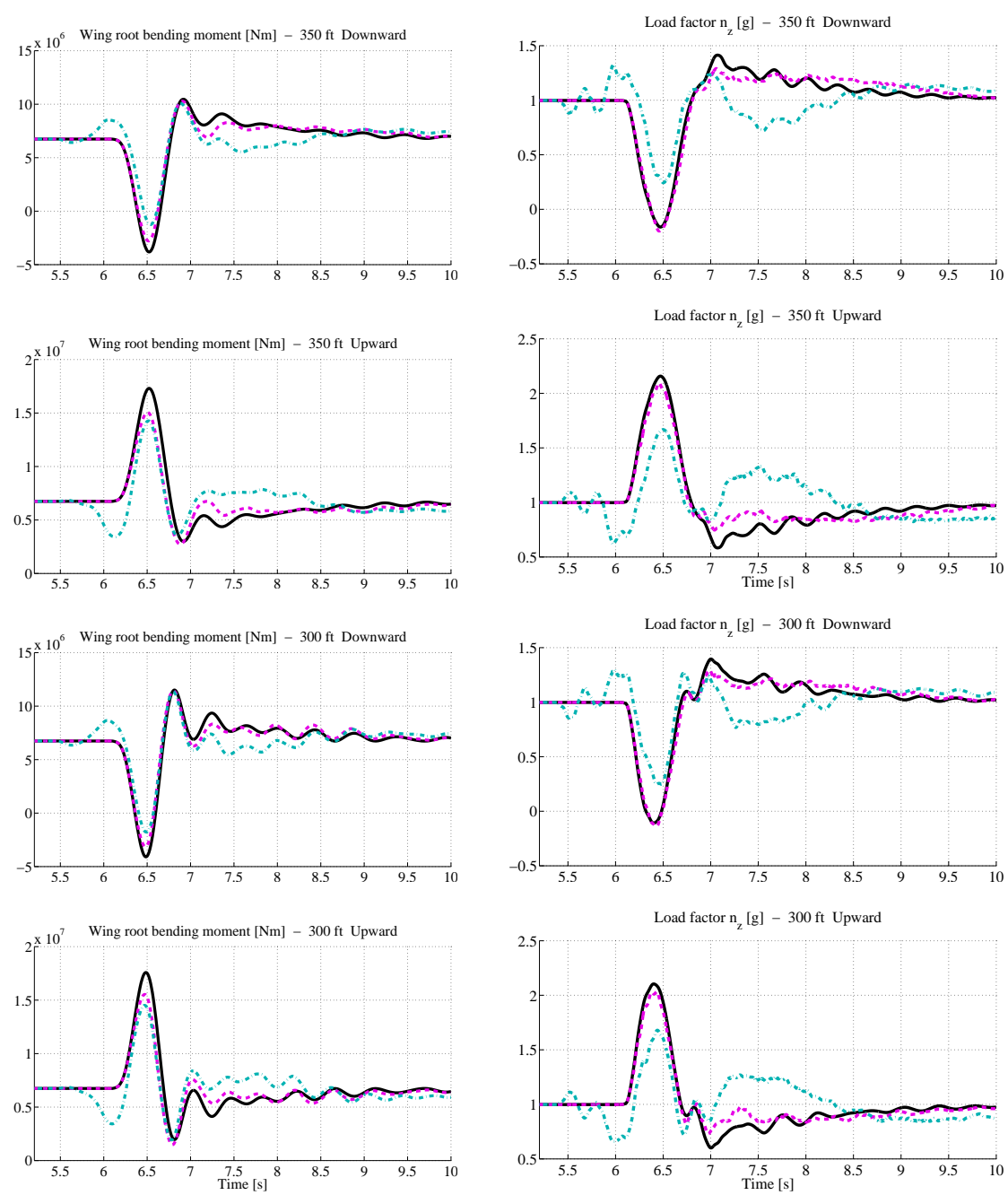

\section{- EFCS}

$\therefore=$ EFCS and FBALC

Fig. 12: Comparison of wing root bending moments (left) and vertical accelerations at middle of cabin (right) over time during encounters with 1 -cosine gusts of lengths 350 and $300 \mathrm{ft}$.

function can easily be seen by the fact that the dash-dotted cyan line begins to vary before the other two: this variation is mainly due to the pitching command that anticipates that loads in the opposite direction are expected to occur very shortly after. In all these simulations the gusts begin at the time $t=6$ seconds. The first seconds are not shown here and are not relevant for the loads analysis: during this time the aircraft flies simply in its trimmed condi- 

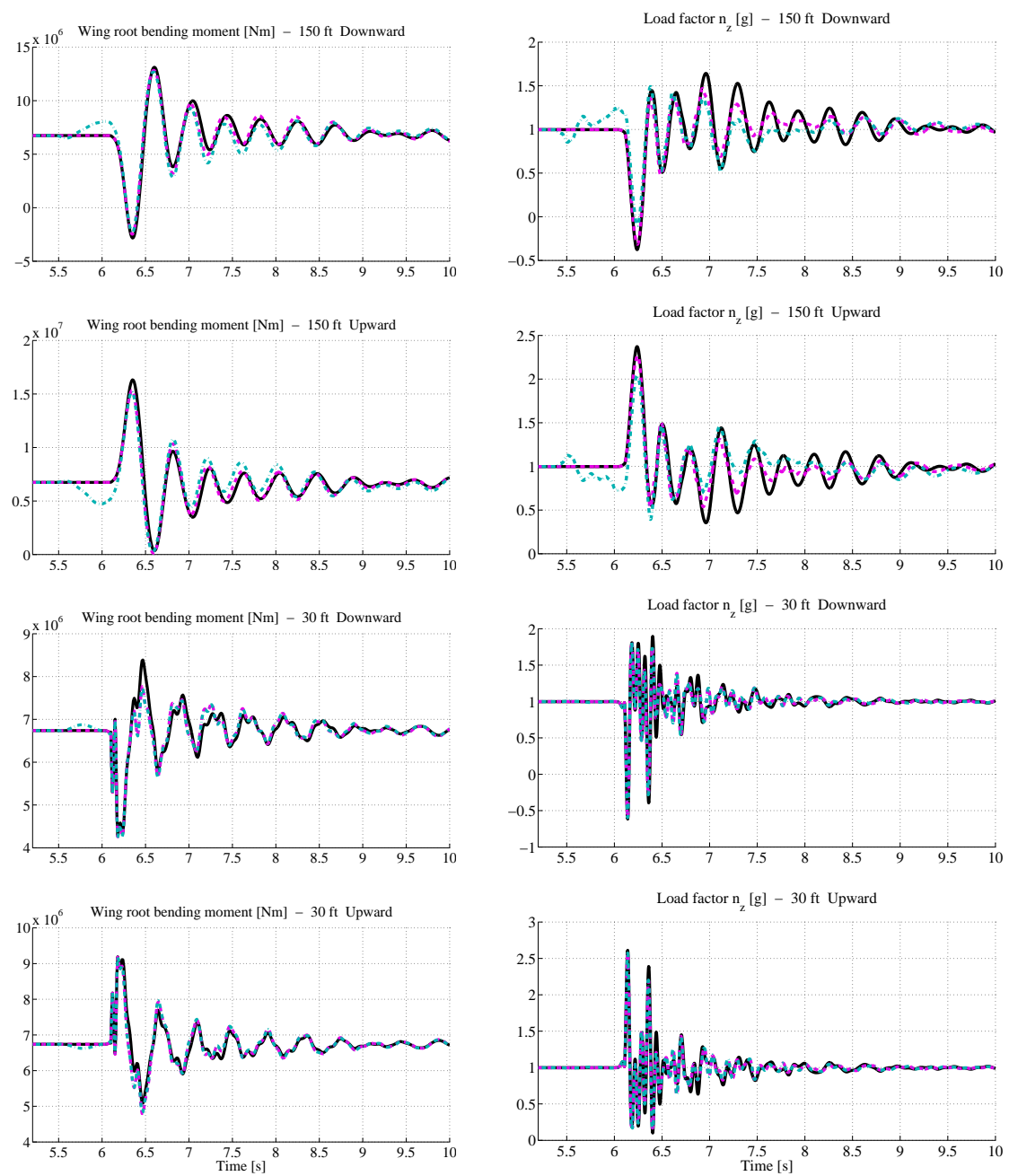

-EFCS

- EFCS and FBALC and GLAREWISE+TFAC

Fig. 13: Comparison of wing root bending moments (left) and vertical accelerations at middle of cabin (right) over time during encounters with 1 -cosine gusts of lengths 150 and $30 \mathrm{ft}$.

tion. These seconds need however to be simulated in order to bring the internal states (e.g. LIDAR measurement database) of the GLAREWISE+TFAC function to a representative state for the various algorithms that are being tested (number of measurements in the buffer, spatial distributions of these measurements, etc.). Note that depending on the sensor and wind reconstruction 
algorithm configurations, six seconds might be more than really necessary for this initialization.

For the $150 \mathrm{ft}$ gust cases (both directions), the FBALC function improves significantly the wing root bending moment and the GLAREWISE+TFAC function only minimally improves it further. For the larger gust lengths $(300 \mathrm{ft}$ and $350 \mathrm{ft}$ ), the load reduction achieved by the FBALC function is also significant, but this time the GLAREWISE + TFAC function achieves a significant additional load reduction. For the $30 \mathrm{ft}$ gusts, the load reductions are very moderate for both "EFCS and FBALC" and "EFCS, FBALC, and GLAREWISE+TFAC" cases. The first peak is even slightly larger if the load alleviation functions are active. Note however, that the reached load levels in the $30 \mathrm{ft}$ cases are very far from the critical loads.

The GLAREWISE+TFAC function also significantly reduces the range of variation of the load factor $n_{z}$, as can be seen on the bottom of figures 12-13. The prevention of relatively long-lasting negative load factors (see downward gusts of lengths $300 \mathrm{ft}$ and $350 \mathrm{ft}$ ) or their significant reduction (see downward gust of length $150 \mathrm{ft}$ ) are likely to be found beneficial by most passengers. The applicability of the commonly used comfort criteria [34,1] to this type of cases is however questionable and therefore these metrics were not used to quantify the comfort on these particular cases. Further investigations aiming at quantifying the impact of the GLAREWISE+TFAC function on comfort should be performed.

Even though both functions (FBALC and GLAREWISE+TFAC) were developed separately and not retuned together yet, they can be combined and provide significant improvements for the longer gust scales. The alleviation performance of the combined feedback/feedforward gust load alleviation function shows promising results already. A significant amount of work shall however still be spent improving both parts and making them work better together. In the presently shown coupling the feedforward function does not really "inform" the feedback function of its actions, which therefore leads the feedback function to consider some of the anticipated actions made by the feedforward controller as "disturbances that should be alleviated". How much additional load alleviation improvement (i.e. on top of the improvement shown here) could be obtained thanks to a better cooperation between both functions is not known and certainly very difficult to estimate. Generally speaking, the evaluation of the performance and the behavior of functions such as the GLAREWISE+TFAC can hardly be done without a complete and fully coupled (atmosphere, aeroelasticity and loads, LIDAR sensor, nonlinear equations of motion, etc.) simulation environment as the one used in this work.

\section{Conclusions and Outlook}

An overview of the active load alleviation activities performed at DLR during the CleanSky Smart Fixed Wing Aircraft project was presented. This work includes two approaches which can also be combined. The first approach is 
based on the use of classical sensors in a feedback scheme and tuned in a multi-objective and multi-model approach. The alleviation performance obtained for all flight points, mass cases, and gust lengths is quite appreciable. Overall the peak and RMS load envelopes are noticeably reduced. The short gusts are too fast to be alleviated by the controller, however they induce only relatively low load levels. The herein presented work focused on Doppler LIDAR measurement processing and exploitation in a feedforward scheme, i.e. all elements of the processing chain that are located between the output of the sensor (line-of-sight measurements) and the load alleviation control surface commands. The obtained improvements were evaluated in a fully coupled simulation environment (generic LIDAR sensor, aeroelastic/loads model, and control functions). The results show a significant improvement compared to the corresponding elements in the demonstrator developed in the AWIATOR project. By combining both functions (feedback and feedforward) a higher performance level is reached compared to the cases where only one of them is used. This work is currently be pursued as part of the CleanSky 2 AirframeITD research framework and considering the application to business jets in addition to the long-range aircraft already considered in this work. The improvement of the wind reconstruction algorithm shown in this paper is one of the first results achieved in this new project.

\section{References}

1. ISO 2631-1:1997: Mechanical vibration and shock - evaluation of human exposure to whole-body vibration - part 1: General requirements (1997)

2. Barbaresco, F.: Airport radar monitoring of wake vortex in all weather conditions. In: European Radar Conference (EuRAD), pp. 85-88 (2010)

3. Barbaresco, F., Meier, U.: Radar monitoring of a wake vortex: Electromagnetic reflection of wake turbulence in clear air. Comptes Rendus Physique 11(1), 54-67 (2010)

4. Böhret, H., Krag, B., Skudridakis, J.: OLGA - An Open-Loop Gust Alleviation System. In: Proceedings of the AGARD CP 384 Meeting. Toronto, Canada (1985)

5. Cézard, N., Besson, C., Dolfi-Bouteyre, A., Lombard, L.: Airflow characterization by Rayleigh-Mie lidars. AerospaceLab 1, 1-4 (2009)

6. Ehlers, J., Fezans, N.: Airborne doppler LiDAR sensor parameter analysis for wake vortex impact alleviation purposes. In: J. Bordeneuve-Guibé, A. Drouin, C. Roos (eds.) Advances in Aerospace Guidance, Navigation and Control, pp. 433-453. Springer (2015)

7. Ehlers, J., Fischenberg, D., Niedermeier, D.: Wake identification based wake impact alleviation control. In: Proceedings of the $14^{\text {th }}$ AIAA Aviation Technology, Integration, and Operations Conference. Atlanta, GA, USA (2014). AIAA-2014-2591

8. Fezans, N.: An unusual structure for a feedforward gust load alleviation controller. In: Proceedings of the 2017 CEAS EuroGNC Conference. Warsaw, Poland (2017)

9. Fezans, N., Joos, H.D.: Integrated active gust and turbulence load alleviation functions. Tech. rep., DLR-IB-111-2014/51 - CleanSky SFWA OP1.2.3-25, DLR, Germany (2014)

10. Fezans, N., Joos, H.D.: Combined feedback and LIDAR-based feedforward active load alleviation. In: AIAA Atmospheric Flight Mechanics Conference, AIAA AVIATION Forum (2017). AIAA-2017-3548, https://doi.org/10.2514/6.2017-3548

11. Fezans, N., Schwithal, J., Fischenberg, D.: In-flight remote sensing and characterization of gusts, turbulence, and wake vortices. In: Proceedings of the 2015 German Aerospace Congress (Deutscher Luft- und Raumfahrtkongress). Rostock, Germany (2015)

12. Fezans, N., Schwithal, J., Fischenberg, D.: In-flight remote sensing and identification of gusts, turbulence, and wake vortices using a Doppler LIDAR. CEAS Aeronautical Journal 8(2) (2017). Doi:10.1007/s13272-017-0240-9 
13. Geisbauer, S., Löser, T.: Towards the investigation of unsteady spoiler aerodynamics. In: 35th AIAA Applied Aerodynamics Conference (2017). URL http://elib.dlr.de/112800/

14. Gerz, T., Holzäpfel, F., Darracq, D.: Commercial aircraft wake vortices. Progress in Aerospace Sciences 38, 181-208 (2002)

15. Hahn, K.U., Hecker, S.: Gust load alleviation system. Tech. rep., DLR, Braunschweig, Germany (2004). AWIATOR, Technical Report, DLR-TR-3.1.1-12

16. Hahn, K.U., König, R.: Attas flight test and simulation results of the advanced gust management system LARS. In: Proceedings of the AIAA Atmospheric Flight Mechanics Conference. Hilton Head, SC, USA (1992)

17. Hargrove, W.J.: The C-5A active lift distribution control system. Tech. rep., NASA (1976). N76-31148

18. Hecker, S.: Proposal of gust load alleviation system using adaptive elements. Tech. rep., DLR, Oberpfaffenhofen, Germany (2005). AWIATOR, Technical Report, DLRTR-3.1.1-13

19. Hecker, S., Hahn, K.U.: Gust computation system. Tech. rep., DLR, Oberpfaffenhofen, Germany (2003). AWIATOR, Technical Report, DLR-TR-3.1.1-11

20. Hecker, S., Hahn, K.U.: Proposal of gust load alleviation system using turbulence sensor and adaptive elements. Tech. rep., DLR, Oberpfaffenhofen, Germany (2006). AWIATOR, Technical Report, DLR-TR-3.1.1-14

21. Herbst, J., Vrancken, P.: Design of a monolithic Michelson interferometer for fringe imaging in a near-field, UV, direct-detection Doppler wind lidar. Applied Optics 55(25), 6910-6929 (2016)

22. Hill, C., Harris, M.: Remote Sensing (UpWind WP6) - QinetiQ Lidar Measurement Report. Tech. Rep. QINETIQ/TS/FPPS/TR0900813, QinetiQ (2010)

23. Hirschberger, M.C.: Beiträge zur Erfassung von Wirbelschleppen mit Lidar - Simulation und Analyse rückgestreuter Signale zur Windfeldbestimmung vor Flugzeugen. Ph.D. thesis, Ludwig-Maximilians-Universität, Munich, Germany (2013)

24. Hoffmann, G.: Stabilisierung, Böenkompensation und Schwingungsdämpfung am elastischen, beweglichen Flugzeugmodell im Windkanal. Tech. Rep. DLR-FB 74-44, DFVLR (now part of DLR), Cologne, Germany (1976)

25. Jeanneau, M., Aversa, N., Delannoy, S., Hockenhull, M.: AWIATOR's Study of a Wing Load Control: Design and Flight-Test Results. In: Proceedings of the $16^{\text {th }}$ IFAC Symposium on Automatic Control in Aerospace. Saint-Petersburg, Russia (2004)

26. Joos, H.D.: Multi-Objective Parameter Synthesis (MOPS). In: J.F. Magni, S. Bennani, J. Terlouw (eds.) Robust Flight Control: A Design Challenge, Lecture notes in control and information sciences, vol. 224, pp. 199-217. Springer (1997)

27. Joos, H.D.: A methodology for multi-objective design assessment and flight control synthesis tuning. Aerospace Science and Technology 3(3), 161-176 (1999)

28. Joos, H.D.: Worst case parameter search based clearance using parallel nonlinear programming methods. In: A. Varga, A. Hansson, G. Puyou (eds.) Optimization Based Clearance of Flight Control Laws, Lecture Notes in Control and Information Science, vol. 416. Springer (2011)

29. Joos, H.D., Bals, J., Looye, G., Schnepper, K., Varga, A.: A multi-objective optimisation based software environment for control systems design. In: Proceedings of the 2002 IEEE International Conference on Control Applications and International Symposium on Computer Aided Control Systems Design (CCA/CACSD). Glasgow, Scotland, U.K. (2002)

30. König, R., Hahn, K.U.: Load alleviation and ride smoothing investigations using ATTAS. In: Proceedings of the $17^{\text {th }}$ Congress of the International Council of the Aeronautical Sciences. Stockholm, Sweden (1990)

31. König, R., Hahn, K.U., Winter, J.: Advanced gust management systems - lessons learned and perspectives. In: Proceedings of the AGARD Flight Mechanics Panel Symposium on Active Control Technology: Applications and Lessons Learned. Torino, Italy (1994)

32. Kordt, M., Ballauf, C., Joos, H.D.: Load alleviation for large aeroplanes by active mode control of the coplanar motion of the horizontal tailplane. In: Proceedings of IFASD International Forum on Aeroelasticity and Structural Dynamics. Madrid, Spain (2001)

33. Krag, B.: The wind tunnel behaviour of a scaled model with a gust alleviation system in a deterministic gust field. Transactions of the Institute of Measurement and Control $\mathbf{1}(3)$ (1979) 
34. Kubica, F., Madelaine, B.: Passenger comfort improvement by integrated control law design. In: Proceedings of the RTO AVT Specialists' Meeting on "Structural Aspects of Flexible Aircraft Control". Ottawa, Canada (1999)

35. Looye, G., Joos, H.D.: Design of robust dynamic inversion control laws using multiobjective optimization. In: Proceedings of the AIAA Guidance, Navigation, and Control Conference and Exhibit. Montreal, Canada (2001). AIAA-2001-4285

36. Looye, G., Joos, H.D., Willemsen, D.: Application of an optimization-based design process for robust autoland control laws. In: Proceedings of the AIAA Guidance, Navigation, and Control Conference and Exhibit. Montreal, Canada (2001)

37. Looye, G., Leitner, M.: XRF-1 model guide. Tech. rep., CleanSky SFWA-ITD D1.2.4.35, DLR, Oberpfaffenhofen, Germany (2013)

38. Luckner, R.: Modeling and simulation of wake vortex encounters: State-of-the-art and challenges. In: Proceedings of the AIAA Modeling and Simulation Technologies Conference. Minneapolis, MN, USA (2012). AIAA-2012-4633

39. Marr, D., Hildreth, E.: Theory of edge detection. Proceedings of the Royal Society of London. Series B, Biological Sciences 207(1167), 187-217 (1980)

40. Neece, R.T., Britt, C.L., White, J.H., Mudukutore, A., Nguyen, C., Hooper, B.: Wake vortex tracking using a $35 \mathrm{GHz}$ pulsed Doppler radar. In: Proceedings of the $5^{\text {th }}$ NASA Integrated Communications, Navigation, and Surveillance (ICNS) Conference and Workshop. Fairfax, VA, USA (2-5 May 2005)

41. Rabadan, G.J., Schmitt, N.P., Pistner, T., Rehm, W.: Airborne lidar for automatic feedforward control of turbulent in-flight phenomena. Journal of Aircraft $\mathbf{4 7}(2), 392$ $403(2010)$

42. Regan, C.D., Jutte, C.V.: Survey of applications of active control technology for gust alleviation and new challenges for lighter-weight aircraft. Tech. rep, TM-2012-216008, NASA, Dryden Flight Research Center, Edwards, CA, USA (2012)

43. Schmitt, N.P., Rehm, W., Pistner, T., Zeller, P., Reithmeier, G., Stilkerich, S., Schertler, K., Diehl, H., Zinner, H.: The AWIATOR airborne LIDAR turbulence sensor. In: Proceedings of the 2005 German Aerospace Congress (Deutscher Luft- und Raumfahrtkongress / DLRK). Friedrichshafen, Germany (2005). DGLR-2005-067

44. Tikhonov, A.N., Arsenin, V.Y.: Solutions of Ill-Posed Problems. Winston and Sons, Washington, DC, USA (1977)

45. Tikhonov, A.N., Goncharsky, A., Stepanov, V.V., Yagola, A.G.: Numerical Methods for the Solution of Ill-Posed Problems. Springer-Science+Business Media, B.V., Dordrecht, The Netherlands (1995). ISBN-13: 978-0-7923-3583-2

46. Vrancken, P.: Airborne remote detection of turbulence with forward-pointing LIDAR. In: R. Sharman, T. Lane (eds.) Aviation Turbulence - Processes, Detection, Prediction, chap. 22, pp. 443-464. Springer (2016). ISBN: 978-3-319-23629-2

47. Vrancken, P., Wirth, M., Ehret, G., Barny, H., Rondeau, P., Veerman, H.: Airborne forward-pointing UV Rayleigh lidar for remote clear air turbulence detection: system design and performance. Applied Optics 55(32), 9314-9328 (2016)

48. Vrancken, P., Wirth, M., Ehret, G., Witschas, B., Veerman, H., Tump, R., Barny H., Rondeau, P., Dolfi-Bouteyre, A., Lombard, L.: Flight tests of the DELICAT airborne LIDAR system for remote clear air turbulence detection. In: 27th International Laser Radar Conference. New York City, NY, USA (2015). Https://doi.org/10.1051/epjconf/201611914003

49. Wolkensinger, C.: Vergleich messtechnischer Konzepte zur bordgestützten Ermittlung atmosphärischer Störphänomene. DLR report DLR-IB-111-2010/35, DLR, Institute of Flight Systems, Braunschweig, Germany (2010)

\title{
Acknowledgements
}

\author{
Most of this work has been funded within the framework of the European CleanSky Joint \\ Technology Initiative - Smart Fixed Wing Aircraft (Grant Agreement Number CSJU-GAM-SFWA- \\ 2008-01) and is currently being pursued within the framework of the European CleanSky2 Joint \\ Technology Initiative - Airframe (Grant Agreement Number CS2JU-AIR-GAM-2014-2015-01 Annex \\ 1, Issue Bo4, October 2nd, 2015) being part of the Horizon 2020 research and Innovation \\ framework programme of the European Commission.
}

The authors would like to thank all the partners of the Smart Fixed Wing Aircraft WP1.2 for the very interesting and open discussions all along the project, Airbus for providing the XRF1 model data, as well as Thiemo Kier for his work on the benchmark model development based on these data. 